TRANSACTIONS OF THE

AMERICAN MATHEMATICAL SOCIETY

Volume 359, Number 7, July 2007, Pages 3125-3151

S 0002-9947(07)04073-1

Article electronically published on February 14, 2007

\title{
IMAGES OF THE BROWNIAN SHEET
}

\author{
DAVAR KHOSHNEVISAN AND YIMIN XIAO
}

\begin{abstract}
An $N$-parameter Brownian sheet in $\mathbf{R}^{d}$ maps a non-random compact set $F$ in $\mathbf{R}_{+}^{N}$ to the random compact set $B(F)$ in $\mathbf{R}^{d}$. We prove two results on the image-set $B(F)$ :

(1) It has positive $d$-dimensional Lebesgue measure if and only if $F$ has positive $\frac{d}{2}$-dimensional capacity. This generalizes greatly the earlier works of J. Hawkes (1977), J.-P. Kahane (1985), and Khoshnevisan (1999).

(2) If $\operatorname{dim}_{\mathcal{H}} F>\frac{d}{2}$, then with probability one, we can find a finite number of points $\zeta_{1}, \ldots, \zeta_{m} \in \mathbf{R}^{d}$ such that for any rotation matrix $\theta$ that leaves $F$ in $\mathbf{R}_{+}^{N}$, one of the $\zeta_{i}$ 's is interior to $B(\theta F)$. In particular, $B(F)$ has interior-points a.s. This verifies a conjecture of T. S. Mountford (1989).

This paper contains two novel ideas: To prove (1), we introduce and analyze a family of bridged sheets. Item (2) is proved by developing a notion of "sectorial local-non-determinism (LND)." Both ideas may be of independent interest.

We showcase sectorial LND further by exhibiting some arithmetic properties of standard Brownian motion; this completes the work initiated by Mountford (1988).
\end{abstract}

\section{INTRODUCTION}

Let $B=\{B(t)\}_{t \in \mathbf{R}_{+}^{N}}$ denote the $(N, d)$-Brownian sheet. That is, $B$ is the $N$ parameter Gaussian random field with values in $\mathbf{R}^{d}$; its mean-function is zero, and its covariance function is given by the following:

$$
\operatorname{Cov}\left(B_{i}(s), B_{j}(t)\right)= \begin{cases}\prod_{k=1}^{N} \min \left(s_{k}, t_{k}\right), & \text { if } 1 \leq i=j \leq d \\ 0, & \text { otherwise }\end{cases}
$$

We have written $B(t)$ in vector form as $\left(B_{1}(t), \ldots, B_{d}(t)\right)$, as is customary.

When $N=1, B$ is just Brownian motion in $\mathbf{R}^{d}$. In this case, it is well known [9] that for any non-random compact set $F \subseteq \mathbf{R}_{+}$,

$$
\mathrm{P}\left\{\lambda_{d}(B(F))>0\right\}>0 \quad \text { if and only if } \operatorname{Cap}_{d / 2}(F)>0 .
$$

Here, $\lambda_{d}$ denotes the $d$-dimensional Lebesgue measure, and for all $\alpha>0, \operatorname{Cap}_{\alpha}(F)$ denotes the $\alpha$-dimensional Bessel-Riesz capacity of $F$ based on the $\alpha$-dimensional

Received by the editors September 12, 2004 and, in revised form, April 21, 2005.

2000 Mathematics Subject Classification. Primary 60G15, 60G17, 28A80.

Key words and phrases. Brownian sheet, image, Bessel-Riesz capacity, Hausdorff dimension, interior-point.

This research was supported by a generous grant from the National Science Foundation.

(C)2007 American Mathematical Society Reverts to public domain 28 years from publication 
energy form $I_{\alpha}$; i.e.,

$$
\operatorname{Cap}_{\alpha}(F)=\left[\inf _{\mu \in \mathscr{P}(F)} I_{\alpha}(\mu)\right]^{-1}, \text { where } I_{\alpha}(\mu)=\iint \frac{\mu(d s) \mu(d t)}{\|s-t\|^{\alpha}},
$$

and $\mathscr{P}(F)$ denotes the collection of all probability measures that are supported in $F$.

According to Taylor's theorem [15, Corollary 2.3.1, p. 525], for all $F \subset \mathbf{R}_{+}^{N}$, $\operatorname{Cap}_{\alpha}(F)=0$ except possibly when $\alpha<N$. Therefore, when $N=1$, (1.2) has non-trivial content when, and only when, $d=1$.

In order to go beyond the one-dimensional case, 10 proposed considering $N$ parameter processes (i.e., fractional Brownian motion), and devised a Fourieranalytic argument which, in the present setting, implies the following for the Brownian sheet:

$$
\mathrm{Cap}_{d / 2}(F)>0 \Longrightarrow \mathrm{P}\left\{\lambda_{d}(B(F))>0\right\}>0 \Longrightarrow \mathrm{H}_{d / 2}(F)>0 .
$$

Here, $\mathrm{H}_{\alpha}$ denotes the $\alpha$-dimensional Hausdorff measure 10, p. 131, Remark 4]. There is an obvious gap between the enveloping conditions of positive capacity and measure. In the special case that $N=2$, this gap was closed in [16, but the problem for $N>2$ has remained open. One of the intentions of this article is to complete the existing picture by deriving the following:

Theorem 1.1. For any choice of $N$ and $d$, and for all non-random compact sets $F$, the d-dimensional Lebesgue measure of $B(F)$ is positive with positive probability if and only if $\operatorname{Cap}_{d / 2}(F)>0$.

We will also prove that the following is an equivalent formulation of Theorem 1.1.

Theorem 1.2. For any choice of $N$ and $d$, for all non-random compact sets $F$, and for every $a \in \mathbf{R}^{d}, \mathrm{P}\left\{B^{-1}(\{a\}) \cap F \neq \varnothing\right\}>0$ if and only if $\operatorname{Cap}_{d / 2}(F)>0$.

On one hand, this and Taylor's theorem together show that if $N \leq d / 2$, then $B^{-1}(\{x\})=\varnothing$ almost surely for all $x \in \mathbf{R}^{d}$. On the other hand, when $N>d / 2$, the codimension of $B^{-1}(\{x\})$ is almost surely $d / 2$ [15, $\S 4.7$, p. 435]. In particular, we can conclude from Theorem 4.7.1 of [15, p. 436] that the Hausdorff-Besicovitch dimension of $B^{-1}(\{x\})$ is a.s. $N-(d / 2)$. When $d=1$, this last assertion is due to Adler [1] $[$. The general case $1 \leq d<2 N$ was treated by [].

In fact, one can go a bit farther at little extra cost. Suppose $f: \mathbf{R}_{+} \rightarrow \mathbf{R}_{+} \cup\{\infty\}$ is a non-increasing measurable function that is finite everywhere except possibly at zero. We can then define the $f$-capacity of a Borel set $F \subseteq \mathbf{R}_{+}^{N}$ as

$$
\operatorname{Cap}_{f}(F)=\left[\inf _{\mu \in \mathscr{P}(F)} I_{f}(\mu)\right]^{-1}, \text { where } I_{f}(\mu)=\iint f(\|s-t\|) \mu(d s) \mu(d t) .
$$

After combining our Theorem 1.2 with Theorem 15.2 of [20], we immediately obtain the following extension of Theorem 5 of $[9]$.

Corollary 1.3. Let $f: \mathbf{R}_{+} \rightarrow \mathbf{R}_{+} \cup\{\infty\}$ be a non-increasing measurable function that is finite on $(0, \infty)$. Then for all $a \in \mathbf{R}^{d}$,

$$
\mathrm{P}\left\{\operatorname{Cap}_{f}\left(B^{-1}(\{a\})\right)>0\right\}>0 \Longleftrightarrow \int_{0}^{1} \frac{f(t) d t}{t^{(d / 2)-N+1}}<\infty .
$$


Our proof of Theorem 1.1 depends on: (i) Ideas from the potential-theory of multiparameter processes that are nowadays considered standard; and (ii) a novel analysis of a class of embedded bridged sheets [also known as pinned Brownian sheets in literature]. We write Theorem 1.1 to not only document it in its definitive form, but to also highlight some of the features of the said bridges. This bridgeanalysis is used in our forthcoming paper with Robert Dalang and Eulalia Nualart to solve an old open problem on the self-intersections of Brownian sheets.

Thanks to the Frostman theorem of potential theory and Theorem 1.1. $\operatorname{dim}_{\mathcal{H}} F>$ $d / 2$ implies that $B(F)$ can have positive Lebesgue measure, whereas $\operatorname{dim}_{\mathcal{H}} F<d / 2$ implies that $B(F)$ almost-surely has zero Lebesgue measure. We plan to prove that much more is true: "If $\operatorname{dim}_{\mathcal{H}} F>d / 2$, then $B(F)$ has interior-points almost surely."

This type of interior-point problem was first studied by [14 in the case of onedimensional Brownian motion $(N=1)$. In this case, Kaufman proved that if $\operatorname{dim}_{\mathcal{H}} F>1 / 2$, then $B(F)$ has interior-points a.s.

Kahane [10,11] and 21] have extended Kaufman's result to symmetric stable Lévy processes and fractional Brownian motion, respectively.

Mountford 18 has considered such interior-point problems for the Brownian sheet, and proved that if $\operatorname{dim}_{\mathcal{H}} F>d / 2$, then $B(\theta F)$ has interior-points a.s. for almost every rotation $\theta F$ of $F$ that is in $\mathbf{R}_{+}^{N 1}$ Moreover, he has conjectured that $B(F)$ has interior-points a.s. [18, p. 184]. We verify this conjecture by proving that the Brownian sheet has the following striking property:

Theorem 1.4. Let $B$ denote the $(N, d)$-Brownian sheet, and let $F \subset \mathbf{R}_{+}^{N}$ be any non-random Borel set that satisfies $\operatorname{dim}_{\mathcal{H}} F>d / 2$. Then there a.s. exist $\zeta_{1}, \ldots, \zeta_{m} \in \mathbf{R}^{d}$ such that for every rotation matrix $\theta$ that leaves $\theta F$ in $\mathbf{R}_{+}^{N}$ we can find an integer $j \in\{1, \ldots, m\}$ such that $\zeta_{j}$ is interior to $B(\theta F)$. In particular, let $\theta$ denote the identity to see that $B(F)$ has interior-points a.s.

In contrast to the Brownian sheet, fractional Brownian motion is always locally non-deterministic [8,21]. This accounts for the differences between the methods of 21], developed for fractional Brownian motion, and the techniques of [18], developed for the Brownian sheet. As part of our arguments, we prove that the Brownian sheet satisfies a type of "sectorial local-non-determinism" (Proposition 4.2); this property leads to a unification of many of the methods developed for the fractional Brownian motion and those for the Brownian sheet. We will show this, anecdotally, by describing an improvement to older results of [19] on self-intersections of images of ordinary Brownian motion.

The rest of this paper is organized as follows. Sections 2 and 3 review briefly the order structure of $\mathbf{R}^{N}$ and the commuting property of the filtrations associated to the Brownian sheet. Sections 4 and 5 describe the correlation structure of the Brownian sheet, sectorial local non-determinism, and a class of bridged sheets. Theorems 1.1 and 1.2 are proved in Sections 6 and 7, respectively. In Section 8, we prove Theorem 1.4 We make further remarks on the images of Brownian motion, and more general Gaussian random fields, in Sections 9 and 10.

Unspecified positive and finite constants are denoted by $A$. They are usually numbered by the equation in which they appear.

\footnotetext{
1 "Almost every rotation" holds with respect to the Haar measure on rotation matrices.
} 


\section{The ORDER STRUCTURE OF $\mathbf{R}^{N}$}

We need to introduce a good deal of notation in order to exploit the various Markov properties of $B$ "in various directions." This is the sole task of the present section.

2.1. The partial orders. There are $2^{N}$ natural partial orders on $\mathbf{R}^{N}$. There is a convenient way to represent them all. Define,

$$
\Pi_{N}=\text { The power set of }\{1, \ldots, N\} \text {. }
$$

Then, each $\pi \in \Pi_{N}$ can be identified with the partial order $\preccurlyeq \pi$ on $\mathbf{R}^{N}$ as follows: For all $a, b \in \mathbf{R}^{N}$,

$$
a \preccurlyeq \pi b \text { iff for all } i=1, \ldots, N, \begin{cases}a_{i} \leq b_{i}, & \text { if } i \in \pi, \\ a_{i} \geq b_{i}, & \text { if } i \notin \pi .\end{cases}
$$

We always write $\preccurlyeq$ in place of the more cumbersome $\preccurlyeq\{1, \ldots, N\}$.

An important feature of the totality $\{\preccurlyeq \pi\}_{\pi \in \Pi_{N}}$ of these partial orders is that together they order $\mathbf{R}^{N}$. By this we mean that for all $a, b \in \mathbf{R}^{N}$, there exists $\pi=\pi(a, b) \in \Pi_{N}$ such that $a \preccurlyeq \pi b$. [Simply, let $\pi(a, b)=\left\{1 \leq i \leq N: a_{i} \leq b_{i}\right\}$.]

2.2. The PO-minimum. Each partial order $\preccurlyeq \pi$ naturally yields a $\pi$-minimum operation $\curlywedge_{\pi}$ which we describe next.

For each point $b \in \mathbf{R}^{N}$, define $S_{b}^{\pi}$ to be its "shadow in the direction $\pi$ "; i.e.,

$$
S_{b}^{\pi}=\left\{a \in \mathbf{R}^{N}: a \preccurlyeq \pi b\right\} .
$$

Then, given $a, b \in \mathbf{R}^{N}$ and a partial order $\pi \in \Pi_{N}$, we define $a \curlywedge_{\pi} b$ to be the unique point whose shadow in the direction $\pi$ is precisely $S_{a}^{\pi} \cap S_{b}^{\pi}$. Let us emphasize the fact that

$$
c \preccurlyeq \pi a, c \preccurlyeq \pi b \Longrightarrow c \preccurlyeq \pi\left(a \curlywedge_{\pi} b\right) .
$$

It is easy to prove that such a point always exists.

Each partial order $\pi \in \Pi_{N}$ on $\mathbf{R}^{N}$ induces $N$ linear orders $\preccurlyeq_{(\pi, 1)}, \ldots, \preccurlyeq_{(\pi, N)}$ on $\mathbf{R}$ via the following:

$$
\preccurlyeq_{(\pi, \ell)}=\left\{\begin{array}{ll}
\leq, & \text { if } \ell \in \pi, \\
\geq, & \text { if } \ell \notin \pi,
\end{array} \quad \forall \ell=1, \ldots, N .\right.
$$

Of course, one obtains only two distinct partial orders this way: $\leq$ and $\geq$. However, in what is to come, the preceding notation will seemlessly do most of the bookkeeping for us.

\section{The Associated Filtrations}

Consider the $\sigma$-algebras

$$
\mathscr{F}_{\pi}(t)=\sigma\left(\{B(s)\}_{s \preccurlyeq{ }_{\pi} t}\right) \quad{ }^{\forall} t \in \mathbf{R}_{+}^{N}, \pi \in \Pi_{N}
$$

Informally speaking, knowing $\mathscr{F}_{\pi}(t)$ amounts to knowing the portion of the Brownian sheet $B$ that corresponds to the values of $s$ in $\mathbf{R}_{+}^{N}$ that are less than $t$ in the partial order $\pi$.

It is not difficult to see that for each partial order $\pi \in \Pi_{N}$, the collection $\mathscr{F}_{\pi}=$ $\left\{\mathscr{F}_{\pi}(t)\right\}_{t \in \mathbf{R}_{+}^{N}}$ is a filtration indexed by $\left(\mathbf{R}_{+}^{N}, \preccurlyeq \pi\right)$; i.e.,

$$
s \preccurlyeq \pi t \Longrightarrow \mathscr{F}_{\pi}(s) \subseteq \mathscr{F}_{\pi}(t) .
$$


We will assume that each filtration $\mathscr{F}_{\pi}$ is complete in the sense that the $\sigma$-algebra $\mathscr{F}_{\pi}(t)$ is complete for every $t \in \mathbf{R}_{+}^{N}$.

For each partial order $\pi \in \Pi_{N}$, we also define $N$ one-parameter families of $\sigma$ algebras, $\mathscr{F}_{\pi}^{1}, \ldots, \mathscr{F}_{\pi}^{N}$, as follows:

$$
\mathscr{F}_{\pi}^{\ell}(r)=\sigma\left(\{B(s)\}_{s_{\ell} \preccurlyeq(\pi, \ell)}\right) \quad{ }^{\forall} r \in \mathbf{R}_{+} .
$$

Note that $\mathscr{F}_{\pi}^{\ell}$ is a filtration of $\sigma$-algebras indexed by $\left(\mathbf{R}_{+}, \preccurlyeq(\pi, \ell)\right.$. Moreover, for all $t \in \mathbf{R}_{+}^{N}, \mathscr{F}_{\pi}(t)=\bigcap_{\ell=1}^{N} \mathscr{F}_{\pi}^{\ell}\left(t_{\ell}\right)$; this fact follows from Proposition 3.1 below.

Following [15. Chapter 1], we say that $\mathscr{F}_{\pi}$ is commuting, if for all times $t \in \mathbf{R}_{+}^{N}$, the $\sigma$-algebras $\mathscr{F}_{\pi}^{1}\left(t_{1}\right), \ldots, \mathscr{F}_{\pi}^{N}\left(t_{N}\right)$ are conditionally independent given $\mathscr{F}_{\pi}(t)$. This is a slightly more general "F4-type" property than the one of [4.

Proposition 3.1. For every $\pi \in \Pi_{N}$, the filtration $\mathscr{F}_{\pi}$ is commuting in the partial order $\pi$; i.e., for all bounded random variables $Z$,

$$
\mathrm{E}\left[Z \mid \mathscr{F}_{\pi}\left(s \curlywedge_{\pi} t\right)\right]=\mathrm{E}\left(\mathrm{E}\left[Z \mid \mathscr{F}_{\pi}(s)\right] \mid \mathscr{F}_{\pi}(t)\right) \quad \text { a.s. }
$$

Thus, commuting filtrations refers to the commutation of the conditional expectation operators.

Proof. For all $\pi \in \Pi_{N}$ and $t \in(0, \infty)^{N}$, we define $\mathcal{I}(t) \in(0, \infty)^{N}$ [coordinatewise] as follows:

$$
\mathcal{I}_{j}(t):= \begin{cases}t_{j}, & \text { if } j \in \pi, \\ 1 / t_{j}, & \text { if } j \notin \pi .\end{cases}
$$

One can think of the map $\mathcal{I}$ as "inversion off of $\pi . "$ It is easy to verify that $\mathcal{I}(\mathcal{I}(t))=t$ for every $t \in(0, \infty)^{N}$.

Now consider the following stochastic process:

$$
W_{\pi}(t)=\frac{B(\mathcal{I}(t))}{\prod_{j \notin \pi} \mathcal{I}_{j}(t)} \quad{ }^{\forall} t \in \mathbf{R}_{+}^{N} .
$$

This is a Brownian sheet, as can be checked by computing covariances. Moreover,

$$
\sigma\left(\left\{W_{\pi}(s)\right\}_{s \preccurlyeq t}\right)=\sigma\left(\{B(\mathcal{I}(s))\}_{s \preccurlyeq t}\right)=\mathscr{F}_{\pi}(\mathcal{I}(t)) .
$$

Since $\mathcal{I}(\mathcal{I}(t))=t$, (3.7) implies that $\sigma\left(\left\{W_{\pi}(s) ; s \preccurlyeq \mathcal{I}(t)\right\}\right)=\mathscr{F}_{\pi}(t)$. Because $\mathcal{I}\left(s \curlywedge_{\pi} t\right)=\mathcal{I}(s) \curlywedge \mathcal{I}(s)$ for all $s, t \in(0, \infty)^{N}$ and the filtration, in the partial order $\preccurlyeq$, of Brownian sheet is commuting [15, Theorem 2.4.1, p. 237], this shows that $\mathscr{F}_{\pi}$ is also commuting.

The preceding leads us to the following useful representation.

Corollary 3.2. For every $\pi \in \Pi_{N}, j=1, \ldots, N$, and $r \in \mathbf{R}_{+}$, define the conditional expectation operator, $\mathscr{E}_{\pi}^{j, r} Y=\mathrm{E}\left[Y \mid \mathscr{F}_{\pi}^{j}(r)\right]\left(Y \in L^{1}(\mathrm{P})\right)$. Then, for all $t \in \mathbf{R}_{+}^{N}$ and for all $\mathrm{P}$-integrable random variables $Z$,

$$
\mathrm{E}\left[Z \mid \mathscr{F}_{\pi}(t)\right]=\mathscr{E}_{\pi}^{11, t_{1}^{\prime}} \ldots \mathscr{E}_{\pi}^{N, t_{N}^{\prime}} Z,
$$

where $\left(t_{1}^{\prime}, \ldots, t_{N}^{\prime}\right)$ denotes an arbitrary non-random permutation of $t=\left(t_{1}, \ldots, t_{N}\right)$. Thus,

$$
\mathrm{E}\left[\sup _{t \in \mathbf{Q}_{+}^{N}}\left(\mathrm{E}\left[Z \mid \mathscr{F}_{\pi}(t)\right]\right)^{2}\right] \leq 4^{N} \mathrm{E}\left[Z^{2}\right]
$$


Proof. To prove the first display, we simply follow along the proof of Theorem

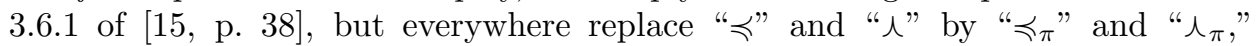
respectively. For the second portion, we apply Doob's strong $(p, p)$-inequality for ordinary martingales $N$ times in succession. For example, see the proof of Cairoli's strong $(p, p)$-inequality [15, Theorem 2.3.1, p. 19], but replace "ß" everywhere by

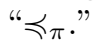

\section{SECTORIAL LOCAL-NON-DETERMINISM}

In this and the next section we state and prove some results on the correlation structure of the Brownian sheet $B=\{B(t)\}_{t \in \mathbf{R}_{+}^{N}}$ in $\mathbf{R}^{d}$. In particular, we prove that $B$ is sectorially locally non-deterministic, and that there is a natural class of bridged sheets associated to $B$. These properties will play an important role in this paper, as well as in studying the self-intersections of the Brownian sheet.

Assumption. Throughout Sections 4 and 5 , we assume that $d=1$.

The following lemma is well known; cf. Lemmas 8.9.1 and 8.9.2 of [1]. For the sake of completeness, we describe a simpler proof.

Lemma 4.1. Choose and fix two numbers $0<a<b<\infty$. If $u, v \in[a, b]^{N}$, then

$$
\frac{a^{N-1}}{\sqrt{N}}\|u-v\| \leq \operatorname{Var}\{B(u)-B(v)\} \leq N b^{N-1}\|u-v\| .
$$

Proof. Let $\sigma(s, t)=\prod_{j=1}^{N} s_{j}-\prod_{j=1}^{N} t_{j}$, and define $s \curlywedge t$ to be the vector whose $i$ th coordinate is $s_{i} \wedge t_{i}$. Then clearly,

$$
\operatorname{Var}\{B(u)-B(v)\}=\sigma(u, u \curlywedge v)+\sigma(v, u \curlywedge v) .
$$

Clearly,

$$
a^{N-1} \max _{1 \leq j \leq N}\left(u_{j}-\left(u_{j} \wedge v_{j}\right)\right) \leq \sigma(u, u \curlywedge v) \leq N b^{N-1} \max _{1 \leq j \leq N}\left(u_{j}-\left(u_{j} \wedge v_{j}\right)\right) .
$$

A similar expression holds for $\sigma(v, u \curlywedge v)$, but everywhere replace $u_{j}-\left(u_{j} \wedge v_{j}\right)$ with $v_{j}-\left(u_{j} \wedge v_{j}\right)$. Add the two series of inequalities, and use the fact that $u_{j}+v_{j}-2\left(u_{j} \wedge v_{j}\right)=\left|u_{j}-v_{j}\right|$ to obtain

$$
a^{N-1} \max _{1 \leq j \leq N}\left|u_{j}-v_{j}\right| \leq \operatorname{Var}\{B(u)-B(v)\} \leq N b^{N-1} \max _{1 \leq j \leq N}\left|u_{j}-v_{j}\right| .
$$

The lemma follows from this and the elementary fact that for all $N$-vectors $x$, $N^{-1 / 2}\|x\| \leq \max _{1 \leq j \leq N}\left|x_{j}\right| \leq\|x\|$.

The Brownian sheet is not locally non-deterministic (LND) with respect to the incremental variance $\operatorname{Var}\{B(u)-B(v)\}$. However, it satisfies the following "sectorial" type of local non-determinism; cf. [15, Lemma 3.3.2, p. 486] for a prefatory version. 
Proposition 4.2 (Sectorial LND). For all positive real number a, integers $n \geq 1$, and all $u, v, t^{1}, \ldots, t^{n} \in[a, \infty)^{N}$,

$$
\begin{aligned}
& \operatorname{Var}\left(B(u) \mid B\left(t^{1}\right), \ldots, B\left(t^{n}\right)\right) \geq \frac{a^{N-1}}{2} \sum_{k=1}^{N} \min _{1 \leq j \leq n}\left|u_{k}-t_{k}^{j}\right|, \\
& \operatorname{Var}\left(B(u)-B(v) \mid B\left(t^{1}\right), \ldots, B\left(t^{n}\right)\right) \\
& \geq \frac{a^{N-1}}{2} \sum_{k=1}^{N} \min \left(\min _{1 \leq j \leq n}\left|u_{k}-t_{k}^{j}\right|+\min _{1 \leq j \leq n}\left|v_{k}-t_{k}^{j}\right|,\left|u_{k}-v_{k}\right|\right) .
\end{aligned}
$$

The proof is divided in two distinct steps. The first is the analysis of the $N=1$ case; we present this portion next.

Lemma 4.3. Let $\{X(t)\}_{t \geq 0}$ denote standard Brownian motion on the line. Then for all times $s, t, s_{1}, \ldots, s_{m} \geq 0$,

$$
\begin{aligned}
\operatorname{Var}(X(s) \mid \mathscr{X}) & \geq \frac{1}{2} \min _{1 \leq j \leq m}\left|s-s_{j}\right|, \\
\operatorname{Var}(X(t)-X(s) \mid \mathscr{X}) & \geq \frac{1}{2} \min \left(\min _{1 \leq j \leq m}\left|s-s_{j}\right|+\min _{1 \leq j \leq m}\left|t-s_{j}\right|,|t-s|\right),
\end{aligned}
$$

where $\mathscr{X}$ denotes the $\sigma$-algebra generated by $\left(X\left(s_{1}\right), \ldots, X\left(s_{m}\right)\right)$.

Proof. Equation (4.7) follows from (4.8). Indeed, let $t=s_{j}$ in (4.8), and then optimize over all $j$ to obtain (4.7).

Equation (4.8) is proved by analyzing two different cases. Throughout, we assume, without any loss of generality, that $s<t$.

Case 1: The first case is where some $s_{j}$ falls between $s$ and $t$. Recall that if $\mathscr{F}$ and $\mathscr{F}^{\prime}$ are linear subspaces (equivalently, $\sigma$-algebras) in the Gauss space $L^{2}(\mathrm{P})$, then for every Gaussian variate $G \in L^{2}(\mathrm{P})$,

$$
\mathscr{F} \subset \mathscr{F}^{\prime} \Longrightarrow \operatorname{Var}(G \mid \mathscr{F}) \geq \operatorname{Var}\left(G \mid \mathscr{F}^{\prime}\right) .
$$

Moreover, both conditional variances are non-random. This elementary fact, used in conjunction with the Markov property, allows us to assume without any further loss in generality that $m=4$ and $s_{1}<s<s_{2}<s_{3}<t<s_{4}$.

Now define $\xi_{1}=X\left(s_{1}\right), \xi_{2}=X(s)-X\left(s_{1}\right), \xi_{3}=X\left(s_{2}\right)-X(s), \xi_{4}=X\left(s_{3}\right)-$ $X\left(s_{2}\right), \xi_{5}=X(t)-X\left(s_{3}\right)$, and $\xi_{6}=X\left(s_{4}\right)-X(t)$. These are independent Gaussian variables, and $\mathscr{X}$ is the linear subspace of $L^{2}(\mathrm{P})$ that is spanned by $\xi_{1},\left(\xi_{1}+\xi_{2}+\xi_{3}\right)$, $\left(\xi_{1}+\xi_{2}+\xi_{3}+\xi_{4}\right)$, and $\left(\xi_{1}+\xi_{2}+\xi_{3}+\xi_{4}+\xi_{5}+\xi_{6}\right)$. Therefore, by the independence of the $\xi_{j}$ 's,

$$
\begin{aligned}
& \operatorname{Var}(X(t)-X(s) \mid \mathscr{X}) \\
& =\operatorname{Var}\left(\xi_{3}+\xi_{5} \mid \xi_{2}+\xi_{3}, \xi_{4}, \xi_{5}+\xi_{6}\right) \\
& =\inf _{\alpha, \beta \in \mathbf{R}} \mathrm{E}\left[\left(\xi_{3}+\xi_{5}-\alpha\left(\xi_{2}+\xi_{3}\right)-\beta\left(\xi_{5}+\xi_{6}\right)\right)^{2}\right] \\
& =\frac{\left(s_{2}-s\right)\left(s-s_{1}\right)}{s_{2}-s_{1}}+\frac{\left(s_{4}-t\right)\left(t-s_{3}\right)}{s_{4}-s_{3}}
\end{aligned}
$$

whence (4.8) in the present case. 
Case 2: The remaining case is where no $s_{j}$ falls in $(s, t)$. In this case, the Markov property shows that we can assume, without loss of generality, that $m=2$ and $s_{1}<s<t<s_{2}$. A direct calculation reveals that in this case,

$$
\operatorname{Var}(X(t)-X(s) \mid \mathscr{X})=\frac{(t-s)\left(s_{2}-s_{1}-t+s\right)}{s_{2}-s_{1}},
$$

from which (4.8) follows.

Proof of Proposition 4.2. Let $\langle a\rangle=(a, \ldots, a)$ designate the lower-left corner of $[a, \infty)^{N}$, and for all $r \geq 0$ and $1 \leq k \leq N$ define

$$
X_{k}(r)=\frac{B(\overbrace{a, \ldots, a}^{k-1 \text { terms }}, a+r, a, \ldots, a)-B(\langle a\rangle)}{a^{(N-1) / 2}} .
$$

The process $\left\{X_{k}(r)\right\}_{r \geq 0}$ is a standard Brownian motion on the line.

For all $t \in[a, \infty)^{N}$, we decompose the rectangle $[0, t]$ into the following disjoint union:

$$
[0, t]=[0, a]^{N} \cup \bigcup_{j=1}^{N} D_{j}\left(t_{j}\right) \cup \Delta(a, t),
$$

where $D_{j}\left(t_{j}\right)=\left\{s \in[0,1]^{N}: 0 \leq s_{i} \leq a\right.$ if $\left.i \neq j, a<s_{j} \leq t_{j}\right\}$ and $\Delta(a, t)$ can be written as a union of $2^{N}-N-1$ sub-rectangles of $[0, t]$. Then we have the following decomposition: For all $t \in[a, \infty)^{N}$,

$$
B(t)=B(\langle a\rangle)+a^{(N-1) / 2} \sum_{k=1}^{N} X_{k}\left(t_{k}-a\right)+B^{\prime}(a, t) .
$$

Here, $B^{\prime}(a, t)=\int_{\Delta(a, t)} d W(s)$ and $W$ is an $N$-parameter Brownian sheet in $\mathbf{R}$ independent of $B$, and all the processes on the right-hand side of (4.14) are independent from one another.

Thus,

$$
\begin{aligned}
& \operatorname{Var}\left(B(u) \mid B\left(t^{1}\right), \cdots, B\left(t^{n}\right)\right) \\
& =\inf _{\alpha \in \mathbf{R}^{n}} \mathrm{E}\left[\left(B(u)-\sum_{j=1}^{n} \alpha_{j} B\left(t^{j}\right)\right)^{2}\right] \\
& \geq a^{N-1} \inf _{\alpha \in \mathbf{R}^{n}} \sum_{k=1}^{N} \operatorname{Var}\left(X_{k}\left(u_{k}-a\right)-\sum_{j=1}^{n} \alpha_{j} X_{k}\left(t_{k}^{j}-a\right)\right) \\
& \geq a^{N-1} \sum_{k=1}^{N} \operatorname{Var}\left(X_{k}\left(u_{k}-a\right) \mid X_{k}\left(t_{k}^{1}-a\right), \ldots, X_{k}\left(t_{k}^{n}-a\right)\right) .
\end{aligned}
$$

Therefore, 4.5) follows from (4.7) and 4.15).

A simple modification of the preceding argument shows that (4.6) follows from (4.8); we omit the details.

We conclude this section with the following result.

Lemma 4.4. Let $n \geq 1$ be a fixed integer. Then for all distinct $t^{1}, \ldots, t^{n} \in(0, \infty)^{N}$, the random variables $B\left(t^{1}\right), \ldots, B\left(t^{n}\right)$ are linearly independent. 
BROWNIAN SHEET

Proof. When all the coordinates of $t^{1}, \ldots, t^{n}$ are distinct, this follows from Proposition 4.2. In general, it suffices to show that for all constants $\alpha_{1}, \ldots, \alpha_{n} \in \mathbf{R}$, if $\operatorname{Var}\left\{\sum_{j=1}^{n} \alpha_{j} B\left(t^{j}\right)\right\}=0$, then $\alpha_{1}=\cdots=\alpha_{n}=0$. The said variance is equal to $\int_{\mathbf{R}_{+}^{N}}\left(\sum_{j=1}^{n} \alpha_{j} \mathbf{1}_{\left[0, t^{j}\right]}(s)\right)^{2} d s$ [15, p. 142], which is assumed to be zero. Thus, $\sum_{j=1}^{n} \alpha_{j} \mathbf{1}_{\left[0, t^{j}\right]}(s)=0$ for a.e. $s \in \mathbf{R}_{+}^{N}$, whence $\alpha_{1}=\cdots=\alpha_{n}=0$.

\section{ANALYSis OF BRIDGES}

For all $s \in \mathbf{R}_{+}^{N}$ we define the process $\left\{B_{s}(t)\right\}_{t \in \mathbf{R}_{+}^{N}}$ as

$$
B_{s}(t)=B(t)-B(s) \prod_{j=1}^{N}\left(\frac{s_{j} \wedge t_{j}}{s_{j}}\right) \quad{ }^{\forall} t \in \mathbf{R}_{+}^{N} .
$$

In the case that $s$ has some coordinates that are zero we define $0 \div 0=1$ to ensure that the preceding is well-defined. Clearly, $B_{s}(s)=0$ and $B_{0}=B$. Thus, the process $B_{s}$ is a realization of the sheet $B$ "conditioned to be zero at time $s . "$ Alternatively, $B_{s}(t)$ is the conditional least-squares estimator of $B(t)$ given $B(s)$; i.e.,

$$
B_{s}(t)=B(t)-\mathrm{E}[B(t) \mid B(s)] .
$$

Hence, for all fixed $s, t \in \mathbf{R}_{+}^{N}, B_{s}(t)$ is independent of $B(s)$. It turns out that much more is true, viz.,

Lemma 5.1. Fix a partial order $\pi \in \Pi_{N}$ and a point $s \in \mathbf{R}_{+}^{N}$. Then,

$$
\left\{B_{s}(t)\right\}_{t_{\succcurlyeq_{\pi} s}} \text { is independent of } \mathscr{F}_{\pi}(s) \text {. }
$$

Proof. Because $B_{s}$ is a Gaussian process it suffices to check that if $t \succcurlyeq_{\pi} s \succcurlyeq_{\pi} u$, then $\operatorname{Cov}\left(B_{s}(t), B(u)\right)=0$. Now

$$
\begin{aligned}
& \operatorname{Cov}(B(t), B(u))=\prod_{j \in \pi} u_{j} \cdot \prod_{j \notin \pi} t_{j}, \\
& \operatorname{Cov}(B(s), B(u))=\prod_{j \in \pi} u_{j} \cdot \prod_{j \notin \pi} s_{j} .
\end{aligned}
$$

The lemma follows because $\prod_{j=1}^{N}\left(s_{j} \wedge t_{j}\right)=\prod_{j \in \pi} s_{j} \cdot \prod_{j \notin \pi} t_{j}$.

Next we prove that the local dynamics of the bridge $\left\{B_{s}(t)\right\}_{t \succcurlyeq_{\pi} s}$ are similar to those of the sheet $B$; compare to Lemma 4.1 .

Lemma 5.2. For each $a>0$, all partial orders $\pi \in \Pi_{N}$, and every $s$, $u$ and $v \in[a, b]^{N}$ that satisfy $s \preccurlyeq_{\pi} u, v$,

$$
\frac{a^{N-1}}{2}\|u-v\| \leq \operatorname{Var}\left\{B_{s}(u)-B_{s}(v)\right\} \leq N b^{N-1}\|u-v\| .
$$

Proof. Thanks to (5.2),

$$
\operatorname{Var}\left\{B_{s}(u)-B_{s}(v)\right\}=\operatorname{Var}(B(u)-B(v) \mid B(s)) .
$$

By (4.9), this is bounded above by $\operatorname{Var}\{B(u)-B(v)\}$, and this expression is at most $N b^{N-1}\|u-v\|$; cf. Lemma 4.1, This proves the upper bound. 
For the lower bound, we apply (4.6) in (5.6) and obtain

$$
\begin{aligned}
\operatorname{Var}\left\{B_{s}(u)-B_{s}(v)\right\} & \geq \frac{a^{N-1}}{2} \sum_{k=1}^{N} \min \left(\left|u_{k}-s_{k}\right|+\left|v_{k}-s_{k}\right|,\left|u_{k}-v_{k}\right|\right) \\
& =\frac{a^{N-1}}{2} \sum_{k=1}^{N}\left|u_{k}-v_{k}\right|,
\end{aligned}
$$

owing to the triangle inequality. The lower bound follows.

Lemma 5.3. Fix two numbers $0<a<b<\infty$. Then there exists a finite constant $A_{5.8}>1$, which depends only on $(N, a, b)$, such that for all $s, t \in[a, b]^{N}$ and all $\varepsilon>0$,

$$
A_{\frac{5.8}{2}}^{-1} \exp \left(-\frac{\varepsilon^{2}}{A_{5.8}\|s-t\|}\right) \cdot \frac{\varepsilon}{\|s-t\|^{1 / 2}} \leq \mathrm{P}\left\{\left|B_{s}(t)\right| \leq \varepsilon\right\} \leq A_{5.8} \frac{\varepsilon}{\|s-t\|^{1 / 2}} .
$$

Proof. We derive the upper bound first:

$$
\begin{aligned}
\mathrm{P}\left\{\left|B_{s}(t)\right| \leq \varepsilon\right\} & =\frac{1}{\sqrt{2 \pi \operatorname{Var} B_{s}(t)}} \int_{-\varepsilon}^{\varepsilon} \exp \left(-\frac{z^{2}}{\operatorname{Var} B_{s}(t)}\right) d z \\
& \leq \varepsilon \sqrt{\frac{2}{\pi \operatorname{Var} B_{s}(t)}} .
\end{aligned}
$$

For the $s$ and $t$ in question we can find a partial order $\pi \in \Pi_{N}$ such that $s \preccurlyeq_{\pi} t$. Therefore, thanks to Lemmas 4.1 and 5.2, we have

$$
\frac{a^{N-1}}{2}\|s-t\| \leq \operatorname{Var} B_{s}(t) \leq N b^{N-1}\|s-t\| .
$$

The upper bound follows. The lower bound is derived similarly.

We end with a final elementary lemma on Gaussian ball-estimates:

Lemma 5.4. Suppose $Y$ is a centered one-dimensional Gaussian random variable with variance $\sigma^{2}$. Let $\alpha$ and $\beta$ be two fixed positive numbers. Then, for all $x \in$ $[-\alpha \sigma,+\alpha \sigma]$,

$$
\mathrm{P}\{|Y-x| \leq \varepsilon\} \geq \begin{cases}e^{-\frac{1}{2} \alpha^{2}-\alpha \beta} \mathrm{P}\{|Y| \leq \varepsilon\}, & \text { if } \varepsilon \leq \beta \sigma, \\ \sqrt{\frac{2}{\pi}} \beta e^{-(\alpha+\beta)^{2} / 2}, & \text { if } \varepsilon>\beta \sigma .\end{cases}
$$

Proof. Evidently,

$$
\begin{aligned}
\mathrm{P}\{|Y-x| \leq \varepsilon\} & =\int_{-\varepsilon}^{\varepsilon} e^{-(z+x)^{2} /\left(2 \sigma^{2}\right)} \frac{d z}{\sigma \sqrt{2 \pi}} \\
& \geq e^{-\frac{1}{2} \alpha^{2}-\alpha \varepsilon / \sigma} \int_{-\varepsilon}^{\varepsilon} e^{-z^{2} /\left(2 \sigma^{2}\right)} \frac{d z}{\sigma \sqrt{2 \pi}} .
\end{aligned}
$$

When $\varepsilon \leq \beta \sigma$, the result follows immediately; when $\varepsilon>\beta \sigma$, use $\int_{-\varepsilon}^{\varepsilon} \geq \int_{-\beta \sigma}^{\beta \sigma}$ in the first line of (5.12), and then change variables $[w:=z / \sigma]$ to deduce the lemma. 


\section{Proof of Theorem 1.1}

6.1. First part. We can first consider $F_{n}=F \cap[1 / n, n]^{N}$, prove the theorem with $F$ replaced by $F_{n}$, and then let $n \uparrow \infty$. This shows that we might as well assume the following:

$$
F \subseteq[a, b]^{N}, \text { where } 0<a<b<\infty .
$$

For all $x \in \mathbf{R}^{d}, \varepsilon>0$, and $\mu \in \mathscr{P}(F)$, define

$$
l_{\mu}^{\varepsilon}(x)=\int \frac{\mathbf{1}_{\{|B(s)-x| \leq \varepsilon\}}}{(2 \varepsilon)^{d}} \mu(d s) .
$$

Also define $p_{t}$ to be the probability density function of $B(t)$; i.e.,

$$
p_{t}(x)=\frac{e^{-\|x\|^{2} /\left(2 \prod_{j=1}^{N} t_{j}\right)}}{\left(2 \pi \prod_{j=1}^{N} t_{j}\right)^{d / 2}} \quad \forall x \in \mathbf{R}^{d}, t \in \mathbf{R}_{+}^{N} .
$$

By Fatou's lemma,

$$
\begin{aligned}
\mathrm{E}\left[l_{\mu}^{\varepsilon}(x)\right] & =(2 \varepsilon)^{-d} \int_{F} \int_{\{|y-x| \leq \varepsilon\}} p_{s}(y) d y \mu(d s) \\
& \geq(1+o(1)) \int p_{s}(x) \mu(d s) \quad(\varepsilon \rightarrow 0) .
\end{aligned}
$$

Thanks to (6.1), we can find a positive and finite constant $A_{6.5}=A_{\overline{6.5}}(N, d, a, b)$, such that for all $\varepsilon \in(0,1), \mu \in \mathscr{P}(F)$, and $x \in \mathbf{R}^{d}$,

$$
\mathrm{E}\left[l_{\mu}^{\varepsilon}(x)\right] \geq A_{\overline{6.5}} \exp \left(-\frac{\|x\|^{2}}{2 A_{\overline{6.5}}}\right) .
$$

Lemma 6.1. Given ([6.1) there exists a positive finite constant $A_{[6.6]}=A_{[6.6}(d, N, a)$ such that for all $x \in \mathbf{R}^{d}, s, t \in \mathbf{R}_{+}^{N}$, and $\varepsilon>0$,

$$
\mathrm{P}\{|B(s)-x| \leq \varepsilon,|B(t)-x| \leq \varepsilon\} \leq A_{\overline{6.6}}\left(\frac{(2 \varepsilon)^{2}}{\|t-s\|^{1 / 2}} \wedge 1\right)^{d} .
$$

Consequently,

$$
\mathrm{E}\left[\left(l_{\mu}^{\varepsilon}(x)\right)^{2}\right] \leq A_{\underline{6.6}} I_{d / 2}(\mu) .
$$

Proof. We will derive (6.6); (6.7) follows from (6.6) and a Fubini-Tonelli argument. We also note that because of the independence of the coordinates, it suffices to prove (6.6) when $d=1$.

Define

$$
C_{s, t}=\prod_{j=1}^{N}\left(\frac{s_{j} \wedge t_{j}}{s_{j}}\right) .
$$

Note that $0 \leq C_{s, t} \leq 1$. Then, recall (5.1) and Lemma 5.1 to deduce that

$$
\begin{aligned}
& \mathrm{P}\{|B(s)-x| \leq \varepsilon,|B(t)-x| \leq \varepsilon\} \\
& =\mathrm{P}\left\{|B(s)-x| \leq \varepsilon,\left|B_{s}(t)+C_{s, t} B(s)-x\right| \leq \varepsilon\right\} \\
& \leq \mathrm{P}\{|B(s)-x| \leq \varepsilon\} \cdot \sup _{z \in \mathbf{R}} \mathrm{P}\left\{\left|B_{s}(t)+z\right| \leq \varepsilon\right\} .
\end{aligned}
$$


Gaussian laws are unimodal, and this means that the supremum is achieved at $z=0$; i.e.,

$$
\mathrm{P}\{|B(s)-x| \leq \varepsilon,|B(t)-x| \leq \varepsilon\} \leq \mathrm{P}\{|B(s)-x| \leq \varepsilon\} \cdot \mathrm{P}\left\{\left|B_{s}(t)\right| \leq \varepsilon\right\} .
$$

Thanks to (6.3) and (6.1),

$$
\mathrm{P}\{|B(s)-x| \leq \varepsilon\}=\int_{x-\varepsilon}^{x+\varepsilon} p_{s}(y) d y \leq 2 \varepsilon p_{s}(0) \leq \varepsilon \sqrt{\frac{2}{\pi a^{N}}} .
$$

On the other hand, by Lemma 5.3, $\mathrm{P}\left\{\left|B_{s}(t)\right| \leq \varepsilon\right\} \leq A_{\overline{5.8}} \varepsilon s-t \|^{-1 / 2}$, whence the lemma.

We are ready to derive half of Theorem 1.1 .

Proof of Theorem 1.1: First half. Thanks to 6.5), Lemma 6.1, and the PaleyZygmund inequality [see, e.g., Kahane [10, p. 8]], for all $\mu \in \mathscr{P}(F)$ and all $\varepsilon \in(0,1)$,

$$
\begin{aligned}
\mathrm{P}\{\operatorname{dist}(x, B(F))<\varepsilon\} & \geq \mathrm{P}\left\{l_{\mu}^{\varepsilon}(x)>0\right\} \geq \frac{\left(\mathrm{E}\left[l_{\mu}^{\varepsilon}(x)\right]\right)^{2}}{\mathrm{E}\left[\left(l_{\mu}^{\varepsilon}(x)\right)^{2}\right]} \\
& \geq \frac{A \sqrt{6.6} \cdot A \sqrt{6.5}}{I_{d / 2}(\mu)} \exp \left(-\frac{\|x\|^{2}}{A \sqrt{6.5}}\right) .
\end{aligned}
$$

The constants on the right-hand side do not depend on $\varepsilon \in(0,1)$ or $\mu \in \mathscr{P}(F)$. Let $\varepsilon \rightarrow 0$ and optimize over $\mu \in \mathscr{P}(F)$ to deduce from the path-continuity of $B$ that the probability of the event $\{x \in B(F)\}$ is at least $A_{6.6} A_{6.5} \exp \left(-\|x\|^{2} / A_{6.5}\right) \operatorname{Cap}_{d / 2}(F)$. Integrate this bound to deduce that whenever $\operatorname{Cap}_{d / 2}(F)>0$, the expected value of $\lambda_{d}(B(F))$ is positive.

Remark 6.2. As we mentioned in the Introduction, we can also use the Fourier analytic method of J.-P. Kahane [10,11] to prove that $\operatorname{Cap}_{d / 2}(F)>0$ implies $\lambda_{d}(B(F))>0$ a.s. The constructive proof in this paper makes it possible to control the value of $\mathrm{E}\left\{\lambda_{d}(B(F))\right\}$ in terms of the capacity $\operatorname{Cap}_{d / 2}(F)$.

6.2. Second part: Step 1. We divide the proof into three steps. In this first step, we derive the main technical inequality which is equation (6.19) below. Throughout this portion of the argument, $\mu$ is an arbitrary probability measure on the fixed compact set $F \subset \mathbf{R}_{+}^{N}$, and $x \in \mathbf{R}^{d}$ is some fixed spatial point. We also choose and fix a partial order $\pi \in \Pi_{N}$ throughout.

Define $C_{s, t}$ by (6.8). Then,

$$
\begin{aligned}
\mathrm{E}\left[l_{\mu}^{\varepsilon}(x) \mid \mathscr{F}_{\pi}(s)\right] & \geq \int_{t \succcurlyeq_{\pi} s} \frac{\mathrm{P}\left\{|B(t)-x| \leq \varepsilon \mid \mathscr{F}_{\pi}(s)\right\}}{(2 \varepsilon)^{d}} \mu(d t) \\
& =\int_{t \succcurlyeq_{\pi} s} \frac{\mathrm{P}\left\{\left|B_{s}(t)+C_{s, t} B(s)-x\right| \leq \varepsilon \mid \mathscr{F}_{\pi}(s)\right\}}{(2 \varepsilon)^{d}} \mu(d t) .
\end{aligned}
$$

Now, as events, we have the obvious inclusion,

$$
\begin{aligned}
& \left\{|B(s)-x|<\frac{\varepsilon}{2}\right\} \cap\left\{\left|B_{s}(t)-\left(1-C_{s, t}\right) x\right| \leq \frac{\varepsilon}{2}\right\} \\
& \subseteq\left\{\left|B_{s}(t)+C_{s, t} B(s)-x\right| \leq \varepsilon\right\} .
\end{aligned}
$$


The preceding two displays together yield the following bound: Almost surely on the event $\{|B(s)-x|<\varepsilon / 2\}$,

$$
\mathrm{E}\left[l_{\mu}^{\varepsilon}(x) \mid \mathscr{F}_{\pi}(s)\right] \geq \int_{t \succcurlyeq_{\pi} s} \frac{\mathrm{P}\left\{\left|B_{s}(t)-\left(1-C_{s, t}\right) x\right| \leq \varepsilon / 2\right\}}{(2 \varepsilon)^{d}} \mu(d t) .
$$

[The conditioning can be removed thanks to Lemma 5.1.]

Next, for all $s \preccurlyeq_{\pi} t$, (6.8) implies

$$
\begin{aligned}
1-C_{s, t} & =\prod_{j \notin \pi} s_{j}^{-1} \cdot\left[\prod_{j \notin \pi} s_{j}-\prod_{j \notin \pi} t_{j}\right] \\
& =\prod_{j \notin \pi} s_{j}^{-1} \cdot \operatorname{Cov}\left(B_{1}(u)-B_{1}(v), B_{1}(u)\right),
\end{aligned}
$$

where $B_{1}$ denotes the first coordinate process of the Brownian sheet $B$, and $u$ and $v$ are defined as follows: For all $j \in \pi, u_{j}=v_{j}=1$ and for all $j \notin \pi, u_{j}=s_{j}$ and $v_{j}=t_{j}$. By the Cauchy-Bunyakovsky-Schwarz inequality, Lemma 4.1, and (6.1),

$$
\begin{aligned}
1-C_{s, t} & \leq \prod_{j \notin \pi} s_{j}^{-1} \cdot \sqrt{\operatorname{Var}\left\{B_{1}(u)-B_{1}(v)\right\} \cdot \operatorname{Var} B_{1}(u)} \\
& \leq \prod_{j \notin \pi} s_{j}^{-1} \cdot \sqrt{N} b^{(N-1) / 2}\|u-v\|^{1 / 2} \prod_{j=1}^{N} u_{j}^{1 / 2} \\
& \leq \sqrt{N} b^{(N-1) / 2}\|s-t\|^{1 / 2} .
\end{aligned}
$$

In particular, we can find a positive and finite constant $A_{\overline{6.18}}=A_{\overline{6.18}}(a, b, d, N)$ such that for all $s, t \in[a, b]^{N}, 1-C_{s, t} \leq A_{[6.18}\|s-t\|^{1 / 2}$. Plug this into (6.15) to obtain the following: Almost surely on the event $\{|B(s)-x|<\varepsilon / 2\}$,

$$
\begin{aligned}
& \mathrm{E}\left[l_{\mu}^{\varepsilon}(x) \mid \mathscr{F}_{\pi}(s)\right]
\end{aligned}
$$

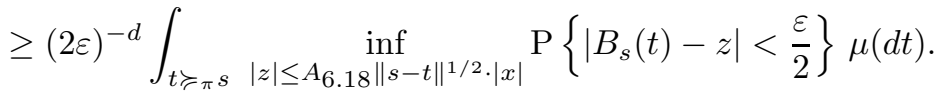

Hold $x \in \mathbf{R}^{d}$ fixed. We can deduce from Lemmas 5.4 and 5.3, and equation (5.10), that there exists a finite constant $A_{[6.19}=A_{6.19}(a, b, d, N, x) \in(0,1)$ such that whenever $\varepsilon \in(0, A \sqrt{6.19})$,

$$
\begin{aligned}
& \mathrm{E}\left[l_{\mu}^{\varepsilon}(x) \mid \mathscr{F}_{\pi}(s)\right] \\
& \geq A_{\frac{6.19}{}} \int_{t \succcurlyeq \pi s}\left[\frac{1}{\max \left(\varepsilon,\|t-s\|^{1 / 2}\right)}\right]^{d} \mu(d t) \cdot \mathbf{1}_{\{|B(s)-x|<\varepsilon / 2\}} .
\end{aligned}
$$

6.3. Step 2. For the second portion of our proof, let us assume that $F$ has a non-empty interior, and of course (6.1) is enforced as well.

We will also make use of the fact that $F$ has a countable dense subset. For simplicity, we assume it is a subset of the rational numbers $\mathbf{Q}_{+}^{N}$. By continuity, the distance between $x$ and $B(F)$ is less than $\varepsilon$ if and only if there exists a rational timepoint $t \in \mathbf{Q}_{+}^{N} \cap F$ such that $\operatorname{dist}(x, B(t))<\varepsilon$. Moreover, the absolute-continuity of the distribution of $B(s)$ - for a given rational time-point $s$ - tells us that the latter happens with positive probability. But it can also happen that with some positive probability $\operatorname{dist}(x, B(t)) \geq \varepsilon$ for all $t \in \mathbf{Q}_{+}^{N} \cap F$. 
In order to properly describe this last assertion, we let $\partial \notin \mathbf{R}_{+}^{N}$ denote a cemetery-point (in time), and define $\mathbf{Q}_{\partial}^{N}=\mathbf{Q}_{+}^{N} \cup\{\partial\}$. Now enumerate all rational time-points to deduce the existence of a $\left(\mathbf{Q}_{\partial}^{N} \cap F\right)$-valued random variable $T_{\varepsilon}$ such that:

(1) $T_{\varepsilon}=\partial$ if and only if $\operatorname{dist}(x, B(F)) \geq \varepsilon$.

(2) On the event $\left\{T_{\varepsilon} \neq \partial\right\}, T_{\varepsilon} \in F$ (a.s.), and $\operatorname{dist}\left(x, B\left(T_{\varepsilon}\right)\right)<\varepsilon$.

Because (6.19) holds almost surely simultaneously for all rational time-points $s$ and all partial orders $\pi \in \Pi_{N}$, it follows that $\sup _{s \in \mathbf{Q}_{+}^{N}}\left|\mathrm{E}\left[l_{\mu}^{\varepsilon}(x) \mid \mathscr{F}_{\pi}(s)\right]\right|^{2}$ is bounded below by

$$
\left(A \overline{6.19} \int_{t \succcurlyeq \pi s}\left[\frac{1}{\max \left(\varepsilon,\left\|T_{\varepsilon / 2}-t\right\|^{1 / 2}\right)}\right]^{d} \mu(d t)\right)^{2} \cdot \mathbf{1}_{\left\{T_{\varepsilon / 2} \neq \partial\right\}} .
$$

So far, everything works for an arbitrary probability measure $\mu$ on $F$. Now we describe a special choice for $\mu$. Namely, we apply the preceding with $\mu$ replaced by $\mu_{\varepsilon} \in \mathscr{P}(F)$, where

$$
\mu_{\varepsilon}(G)=\mathrm{P}\left\{T_{\varepsilon / 2} \in G \mid T_{\varepsilon / 2} \neq \partial\right\} \quad \forall \text { Borel sets } G \subseteq \mathbf{R}_{+}^{N} .
$$

Integrate (6.20) $[d \mathrm{P}]$ to conclude that

$$
\begin{aligned}
& \mathrm{E}\left(\sup _{s \in \mathbf{Q}_{+}^{N}}\left|\mathrm{E}\left[l_{\mu_{\varepsilon}}^{\varepsilon}(x) \mid \mathscr{F}_{\pi}(s)\right]\right|^{2}\right) \\
& \geq \mathrm{E}\left[\left(A_{\left[\frac{6.19}{}\right.} \int_{t \succcurlyeq \pi s}\left[\frac{1}{\max \left(\varepsilon,\left\|T_{\varepsilon / 2}-t\right\|^{1 / 2}\right)}\right]^{d} \mu_{\varepsilon}(d t)\right)^{2} \cdot \mathbf{1}_{\left\{T_{\varepsilon / 2} \neq \partial\right\}}\right] \\
& =A_{[6.19}^{2} \int\left(\int_{t \succcurlyeq_{\pi} s}\left[\frac{1}{\|s-t\|^{1 / 2}} \wedge \frac{1}{\varepsilon}\right]^{d} \mu_{\varepsilon}(d t)\right)^{2} \mu_{\varepsilon}(d s) \cdot \mathrm{P}\left\{T_{\varepsilon / 2} \neq \partial\right\} \\
& \geq A_{[6.19}^{2}\left(\iint_{t \succcurlyeq_{\pi} s}\left[\frac{1}{\|s-t\|^{1 / 2}} \wedge \frac{1}{\varepsilon}\right]^{d} \mu_{\varepsilon}(d t) \mu_{\varepsilon}(d s)\right)^{2} \cdot \mathrm{P}\left\{T_{\varepsilon / 2} \neq \partial\right\} \\
& :=A_{[6.19}^{2} \mathscr{Q}_{\varepsilon, \pi}^{2} \cdot \mathrm{P}\left\{T_{\varepsilon / 2} \neq \partial\right\} .
\end{aligned}
$$

[In the fourth line, we have appealed to the Cauchy-Bunyakovsky-Schwarz inequality.] On the other hand,

$$
\begin{aligned}
& \mathrm{E}\left(\sup _{s \in \mathbf{Q}_{+}^{N}}\left|\mathrm{E}\left[l_{\mu_{\varepsilon}}^{\varepsilon}(x) \mid \mathscr{F}_{\pi}(s)\right]\right|^{2}\right) \\
& \leq 4^{N} \sup _{s \in \mathbf{Q}_{+}^{N}} \mathrm{E}\left(\left|\mathrm{E}\left[l_{\mu_{\varepsilon}}^{\varepsilon}(x) \mid \mathscr{F}_{\pi}(s)\right]\right|^{2}\right) \\
& \leq 4^{N} \mathrm{E}\left[\left(l_{\mu_{\varepsilon}}^{\varepsilon}(x)\right)^{2}\right] \\
& \leq A_{6.23]} \iint\left[\frac{1}{\|s-t\|^{1 / 2}} \wedge \frac{1}{\varepsilon}\right]^{d} \mu_{\varepsilon}(d t) \mu_{\varepsilon}(d s) \\
& =A_{6.23]} \mathscr{Q}_{\varepsilon} .
\end{aligned}
$$


Brief justification: The first line follows from Corollary 3.2 and the third line follows from (6.6) and the Fubini-Tonelli theorem. We re-emphasize that the constants $A_{\overline{6.19}}$ and $A_{\overline{6.23}}$ do not depend on $\varepsilon$ or $\pi$. Add the preceding over all $\pi \in \Pi_{N}$ to obtain

$$
\begin{aligned}
2^{N} A_{[.23]} \mathscr{Q}_{\varepsilon} & \geq A_{[6.19}^{2} \sum_{\pi \in \Pi_{N}} \mathscr{Q}_{\varepsilon, \pi}^{2} \cdot \mathrm{P}\left\{T_{\varepsilon / 2} \neq \partial\right\} \\
& \geq 2^{1-N} A_{6.19}^{2}\left(\sum_{\pi \in \Pi_{N}} \mathscr{Q}_{\varepsilon, \pi}\right)^{2} \cdot \mathrm{P}\left\{T_{\varepsilon / 2} \neq \partial\right\} \\
& \geq 2^{1-N} A_{6.19}^{2} \mathscr{Q}_{\varepsilon}^{2} \cdot \mathrm{P}\left\{T_{\varepsilon / 2} \neq \partial\right\} .
\end{aligned}
$$

Solve for the probability, using the fact that $\mathscr{Q}_{\varepsilon}$ is strictly positive, to obtain

$$
\mathrm{P}\{\operatorname{dist}(x, B(F))<\varepsilon / 2\} \leq \frac{2^{2 N-1} A \overline{6.23}}{A_{\frac{6.19}{2}}^{2} \iint\left[\frac{1}{\|s-t\|^{1 / 2}} \wedge \frac{1}{\varepsilon}\right]^{d} \mu_{\varepsilon}(d t) \mu_{\varepsilon}(d s)} .
$$

Now, $\left\{\mu_{\varepsilon}\right\}_{\varepsilon \in(0,1)}$ is a collection of probability measures on the compact set $F$; let $\mu_{0}$ denote any (weak) limit-measure. Then, $\mu_{0}$ is also a probability measure on $F$, and by the Fatou lemma and the path-continuity of $B$,

$$
\mathrm{P}\{x \in B(F)\} \leq \frac{2^{2 N-1} A_{[6.23}}{A_{[6.19}^{2} I_{d / 2}\left(\mu_{0}\right)},
$$

and this is valid even if $\mu_{0}$ has infinite $(d / 2)$-dimensional energy as long as we interpret $1 \div \infty$ as zero.

6.4. Step 3. If $F$ has an interior, then (6.26) provides us with a hitting estimate; we note once more that $A_{6.19}$ and $A_{\overline{6.23}}$ of the latter equation depend only on $(d, a, b, N, x)$. For a general compact set $F \subseteq[a, b]^{N}$, and given $\eta \in(0,1)$, let $F^{\eta}$ denote the closed $\eta$-enlargement of $F$. Equation (6.26) provides us with a positive finite constant $A_{*}=A_{*}(a, b, d, N, x)$ and a probability measure $\mu^{\eta}$, on $F^{\eta}$, such that

$$
\mathrm{P}\left\{x \in B\left(F^{\eta}\right)\right\} \leq \frac{A_{*}}{I_{d / 2}\left(\mu^{\eta}\right)} .
$$

By the Fatou lemma and weak compactness, we can find a probability measure $\mu_{*}$ on $F$ such that $\liminf _{\eta \rightarrow 0} I_{d / 2}\left(\mu^{\eta}\right) \geq I_{d / 2}\left(\mu_{*}\right)$. Because $A_{*}$ does not depend on $\eta$, path-continuity of $B$ shows that

$$
\mathrm{P}\{x \in B(F)\} \leq \frac{A_{*}}{I_{d / 2}\left(\mu_{*}\right)} \leq A_{*} \operatorname{Cap}_{d / 2}(F) .
$$

In particular, if $F$ has zero $(d / 2)$-dimensional capacity, then $\mathrm{P}\{x \in B(F)\}=0$ for all $x$. A final appeal to the Fubini-Tonelli theorem demonstrates that in this case, the expectation of $\lambda_{d}(B(F))$ is zero. This completes our proof.

\section{Proof of Theorem 1.2}

Our proof of Theorem 1.1 contains the proof of Theorem 1.2, cf. (6.12) and (6.26). However, for the sake of future use we prove the following more general result. It extends some results of Kahane [11,12] on stable Lévy processes. 
Proposition 7.1. Let $B=\{B(t)\}_{t \in \mathbf{R}_{+}^{N}}$ denote the $(N, d)$ Brownian sheet. Let $E \subset \mathbf{R}^{d}$ and $F \subset(0, \infty)^{N}$ be fixed Borel sets. Then the following are equivalent:

(1) With positive probability, $F \cap B^{-1}(E) \neq \varnothing$.

(2) With positive probability, $E \cap B(F) \neq \varnothing$.

(3) With positive probability, $\lambda_{d}(E \ominus B(F))>0$, where for any subsets $E$ and $G$ of $\mathbf{R}^{d}, E \ominus G=\{x-y: x \in E, y \in G\}$.

Proof. Items (1) and (2) are manifestly equivalent. To prove that $(2) \Leftrightarrow(3)$ we may note that (2) is equivalent to the following:

$$
{ }^{\exists} \delta>0 \text { such that } \mathrm{P}\left\{E \cap B\left(F \cap(\delta, \infty)^{N}\right) \neq \varnothing\right\}>0 .
$$

Hence, without loss of generality, we can assume that $F \subset(\delta, \infty)^{N}$ for some $\delta>0$. Fix a $\tau \in(0, \delta)^{N}$ so that $\tau \preccurlyeq t$ for all $t \in F$. Define the random field $B^{\tau}=$ $\left\{B^{\tau}(t)\right\}_{t \in \mathbf{R}_{+}^{N}}$ by

$$
B^{\tau}(t)=B(\tau+t)-B(\tau) \quad{ }^{\forall} t \in \mathbf{R}_{+}^{N}
$$

Observe that

$$
E \cap B(F)=\varnothing \Longleftrightarrow B(\tau) \notin E \ominus B^{\tau}(F-\tau) .
$$

Because $B(\tau)$ is independent of the random Borel set $E \ominus B^{\tau}(F-\tau)$ and the distribution of $B(\tau)$ is equivalent to $\lambda_{d}$, we have

$$
B(\tau) \notin E \ominus B^{\tau}(F-\tau) \quad \text { a.s. } \quad \Longleftrightarrow \lambda_{d}\left(E \ominus B^{\tau}(F-\tau)\right)=0 \quad \text { a.s. }
$$

Note that $B^{\tau}(F-\tau)=B(F) \ominus\{B(\tau)\}$, so that the translation-invariance of the Lebesgue measure, (7.3), and (7.4) together imply that

$$
E \cap B(F)=\varnothing \quad \text { a.s. } \Longleftrightarrow \lambda_{d}(E \ominus B(F))=0 \quad \text { a.s. }
$$

This proves the equivalence of (2) and (3), whence the proposition.

\section{Proof of Theorem 1.4}

Our proof of Theorem 1.4 relies on developing moment-estimates for the local times of the Brownian sheet on $F$, as well as a Fourier-analytic argument. Our argument is closely-related to the methods of [14, [10, [18], and [24].

8.1. First reduction. Without loss of generality, we can [and will] assume that $F$ is compact. Otherwise, we can consider a compact subset $F^{\prime} \subseteq F$ such that $\operatorname{dim}_{\mathcal{H}} F^{\prime}>\frac{d}{2}$; see [7, Theorem 4.10]. Because $B\left(F^{\prime}\right) \subseteq B(F)$, this proves that there is no harm in assuming that (6.1) holds for some $0<a<b$. This compactness assumption on $F$ is in force throughout this section.

Because we have assumed that $\operatorname{dim}_{\mathcal{H}} F>\frac{d}{2}$, we can choose a $\gamma \in(0,1)$ such that

$$
\operatorname{dim}_{\mathcal{H}} F>\frac{\gamma+d}{2} \text {. }
$$

Then by Frostman's lemma, there exists a probability measure $\mu$ on $F$ such that

$$
A_{\overline{8.2}}=\sup _{s \in \mathbf{R}^{N}} \int \frac{\mu(d t)}{\|s-t\|^{(\gamma+d) / 2}}<\infty .
$$

See [10, p. 130] or [15, p. 517]. 
8.2. Second reduction. Fix some $c>0$ and define

$$
F_{\ell}=\left\{t=\left(t_{1}, \ldots, t_{N}\right) \in F: t_{\ell}=c\right\}, \quad \ell=1, \ldots, N .
$$

Suppose there exists $\ell \in\{1, \ldots, N\}$ such that $\mu\left(F_{\ell}\right)>0$, where $\mu$ is the measure that satisfies (8.2). By Frostman's lemma, the Hausdorff dimension of $F_{\ell}$ is strictly greater than $(d / 2)$. Identify $F_{\ell}$ with a set in $\mathbf{R}_{+}^{N-1}$ (ignore the $\ell$ th coordinate), and denote the set in $\mathbf{R}_{+}^{N-1}$ by $F_{\ell}^{\prime}$. The preceding development, and Frostman's lemma, together prove that $\operatorname{dim}_{\mathcal{H}}\left(F_{\ell}^{\prime}\right)>(d / 2)$. It then suffices to prove that $\tilde{B}\left(F_{\ell}^{\prime}\right)$ has an interior point, where $\tilde{B}$ is the $(N-1)$-parameter Brownian sheet in $\mathbf{R}^{d}$. Therefore, we may assume - without loss of generality - that the probability measure $\mu$ of (8.2) has the following property: For all $c>0$ and $\ell=1, \ldots, N$,

$$
\mu\left\{t=\left(t_{1}, \ldots, t_{N}\right) \in F: t_{\ell}=c\right\}=0 .
$$

Now consider the push-forward $\mu \circ B^{-1}$ of $\mu$ by $B$. If $\mu \circ B^{-1} \ll \lambda_{d}$, then $B$ is said to have a local time on $F$. The local time $l_{\mu}(x)$ is defined as the RadonNikodým derivative $d\left(\mu \circ B^{-1}\right) / d \lambda_{d}$ at $x \in \mathbf{R}^{d}$. Another way of writing this is: If $f: \mathbf{R}^{d} \rightarrow \mathbf{R}_{+}$is Borel measurable, then with probability one,

$$
\int_{F} f(B(s)) \mu(d s)=\int_{\mathbf{R}^{d}} f(x) l_{\mu}(x) d x .
$$

It is well known that (8.2) implies that $l_{\mu}$ is in $L^{2}\left(\lambda_{d}\right)$ almost surely; cf. 88, Theorem 22.1] or [10, Theorem 4, p. 204]. In fact, $l_{\mu}$ is the $L^{2}\left(\mathrm{P} \times \lambda_{d}\right)$-limit of $l_{\mu}^{\varepsilon}$ as $\varepsilon$ tends to 0 ; cf. 6.2 .

8.3. Continuity in the space variable. Note that $B(F)$ is compact and $\{x$ : $\left.l_{\mu}(x)>0\right\}$ is a subset of $B(F)$. Hence, in order to prove that $B(F)$ has interiorpoints, it suffices to demonstrate that $l_{\mu}(x)$ has a version which is continuous in $x[8$, p. $12 ; 21$, p. 324]. We are going to have to do more to prove the uniform result for $\theta F$, but for now, we concentrate on $\theta$ being equal to the identity matrix.

Theorem 8.1. Let $F$ be a compact set in $\mathbf{R}_{+}^{N}$ that satisfies (6.1), and suppose $\mu \in \mathscr{P}(F)$ satisfies (8.2). Then for every even integer $n \geq 2$, there exists a finite constant $A_{8.6}$ that depends only on $\left(a, b, d, N, \gamma, A_{8.2}, n\right)$ - such that

$$
\mathrm{E}\left[\sup _{\substack{u, v \in \mathbf{R}^{d}: \\ u \neq v}} \frac{\left|l_{\mu}(u)-l_{\mu}(v)\right|^{n}}{|u-v|^{\lfloor n /(N+1)\rfloor \gamma}}\right] \leq A_{\overline{8.6},}
$$

where $\lfloor x\rfloor$ denotes 1 if $x \leq 1$, and the largest integer $\leq x$ if $x>1$. Consequently, there exists a version of $\left\{l_{\mu}(x)\right\}_{x \in \mathbf{R}^{d}}$ that is uniformly Hölder-continuous with index $\eta$ for any $\eta$ that satisfies

$$
0<\eta<\min \left(1, \frac{2}{N+1}\left(\operatorname{dim}_{\mathcal{H}} F-\frac{d}{2}\right)\right) .
$$

Before proving Theorem 8.1 , we develop two technical lemmas.

For the first lemma, define

$$
\widetilde{F}=\left\{\bar{v}=\left(v^{1}, \ldots, v^{n}\right) \in F^{n} ; v_{\ell}^{j}=v_{\ell}^{i} \text { for some } i \neq j \text { and } 1 \leq \ell \leq N\right\} .
$$


Lemma 8.2. If $\mu \in \mathscr{P}(F)$ satisfies (8.4), then the set $\widetilde{F}$ is $\mu^{n}$-null, where $\mu^{n}=$ $\mu \times \cdots \times \mu$ (n times $)$.

Proof. This follows from (8.4) and the Fubini-Tonelli theorem.

Lemma 8.3. Let $\left\{Z_{i}\right\}_{i=1}^{n}$ be linearly-independent centered Gaussian variables. If $g: \mathbf{R} \rightarrow \mathbf{R}_{+}$is Borel measurable, then

$$
\int_{\mathbf{R}^{n}} g\left(v_{1}\right) e^{-\frac{1}{2} \operatorname{Var}(v \cdot Z)} d v=\frac{(2 \pi)^{(n-1) / 2}}{Q^{1 / 2}} \int_{-\infty}^{\infty} g\left(z / \sigma_{1}\right) e^{-\frac{1}{2} z^{2}} d z
$$

where $\sigma_{1}^{2}=\operatorname{Var}\left(Z_{1} \mid Z_{2}, \ldots, Z_{n}\right)$, and $Q=\operatorname{det} \operatorname{Cov}\left(Z_{1}, \ldots, Z_{n}\right)$ denotes the determinant of the covariance matrix of $Z$.

Proof. In the case that $g$ is bounded follows from [5, Lemma 2]. To prove the general case, replace $g$ by $g \wedge k$ and let $k$ tend to infinity.

We will use the following elementary formula to estimate the determinant of the covariance matrix of a Gaussian vector $Z$ :

$$
\operatorname{det} \operatorname{Cov}\left(Z_{1}, \ldots, Z_{n}\right)=\operatorname{Var}\left(Z_{1}\right) \prod_{j=2}^{n} \operatorname{Var}\left(Z_{j} \mid\left\{Z_{i}\right\}_{i \leq j-1}\right) .
$$

We are ready to present the following.

Proof of Theorem 8.1. By the Fourier inversion theorem, for every $x, y \in \mathbf{R}^{d}$, and all even integers $n \geq 2$,

$$
\begin{aligned}
& \mathrm{E}\left[\left(l_{\mu}(x)-l_{\mu}(x+y)\right)^{n}\right] \\
& =(2 \pi)^{-n d} \int_{F^{n}} \int_{\mathbf{R}^{n d}} \prod_{j=1}^{n}\left[e^{-i u^{j} \cdot x}-e^{-i u^{j} \cdot(x+y)}\right] \\
& \quad \times e^{-\frac{1}{2} \operatorname{Var}\left(\sum_{j=1}^{n} u^{j} \cdot B\left(t^{j}\right)\right)} d \bar{u} \mu^{n}(d \bar{t}) .
\end{aligned}
$$

Here, $\bar{u}=\left(u^{1}, \ldots, u^{n}\right), \bar{t}=\left(t^{1}, \ldots, t^{n}\right)$, and for each $j, u^{j}$ and $t^{j}$ are respectively in $\mathbf{R}^{d}$ and $(0, \infty)^{N}$. The details that lead to (8.11) are explained in [8, Eq. 25.7]; see also 21].

Consider the non-decreasing function $\Lambda(u)=\inf \left\{1,|u|^{\gamma}\right\}$ and the elementary inequality

$$
\left|e^{i u}-1\right| \leq 2 \Lambda(u) \quad \forall u \in \mathbf{R} .
$$

This is valid because $\gamma$ is in $(0,1)$. By the triangle inequality,

$$
\left|e^{-i u^{j} \cdot x}-e^{-i u^{j} \cdot(x+y)}\right| \leq \sum_{k=1}^{d}\left|e^{-i u_{k}^{j} y_{k}}-1\right| .
$$

By expanding the product in (8.11), using (8.13) and (8.12), we obtain

$$
\begin{aligned}
& \mathrm{E}\left[\left(l_{\mu}(x)-l_{\mu}(x+y)\right)^{n}\right] \\
& \leq(2 \pi)^{-n d} 2^{n} \sum^{\prime} \int_{F^{n}} \underbrace{\int_{\mathbf{R}^{n d}} \prod_{j=1}^{n} \Lambda\left(u_{k_{j}}^{j} y_{k_{j}}\right) e^{-\frac{1}{2} \operatorname{Var}\left(\sum_{i=1}^{n} u^{i} \cdot B\left(t^{i}\right)\right)} d \bar{u}}_{:=\mathscr{M}=\mathscr{M}(\bar{k}, \bar{t})} \mu^{n}(d \bar{t}) .
\end{aligned}
$$


Here, $\sum^{\prime}$ signifies the sum over all sequences $\bar{k}=\left(k_{1}, \ldots, k_{n}\right) \in\{1, \ldots, d\}^{n}$. In accordance with Lemma 8.2, the outer integral in (8.14) can be taken to be over $F^{n} \backslash \widetilde{F}$.

Fix $\bar{k}=\left(k_{1}, \ldots, k_{n}\right) \in\{1, \ldots, d\}^{n}$ and $t^{1}, \ldots, t^{n} \in F$; we proceed to estimate the integral $\mathscr{M}$ in (8.14). We will assume that $t_{\ell}^{j}(1 \leq j \leq n, 1 \leq \ell \leq N)$ are distinct (Lemma 8.2). Lemma 4.4 implies that the Gaussian random variables $\left\{B_{k}\left(t^{j}\right) ; k=\right.$ $1, \ldots, d, j=1, \ldots, n\}$ are linearly independent. Hence, by applying the generalized Hölder inequality, Lemma 8.3 , and the independence of the coordinate-processes $B_{1}, \ldots, B_{d}$ of $B$, the quantity $\mathscr{M}$ is bounded above by

$$
\begin{aligned}
\prod_{j=1}^{n}\left\{\int_{\mathbf{R}^{n d}} \Lambda^{n}\left(u_{k_{j}}^{j} y_{k_{j}}\right) e^{-\frac{1}{2} \operatorname{Var}\left(\sum_{i=1}^{n} \sum_{\ell=1}^{d} u_{\ell}^{i} B_{\ell}\left(t^{i}\right)\right)} d \bar{u}\right\}^{1 / n} \\
=\frac{(2 \pi)^{(n d-1) / 2}}{\left[\operatorname{det} \operatorname{Cov}\left(B_{1}\left(t^{1}\right), \ldots, B_{1}\left(t^{n}\right)\right)\right]^{d / 2}} \\
\quad \times \prod_{j=1}^{n}\left\{\int_{-\infty}^{\infty} \Lambda^{n}\left(\frac{y_{k_{j}}}{\sigma_{j}(\bar{t})} z\right) e^{-\frac{1}{2} z^{2}} d z\right\}^{1 / n} .
\end{aligned}
$$

Here, $\sigma_{j}^{2}(\bar{t})$ is the conditional variance of $B_{k_{j}}\left(t^{j}\right)$ given $B_{\ell}\left(t^{i}\right)\left(\ell \neq k_{j}\right.$ and $1 \leq i \leq n$, or $\ell=k_{j}$ and $i \neq j$ ).

Since $B_{1}, \ldots, B_{d}$ are i.i.d., we have

$$
\sigma_{j}^{2}(\bar{t})=\operatorname{Var}\left(B_{1}\left(t^{j}\right) \mid\left\{B_{1}\left(t^{i}\right)\right\}_{i \neq j}\right) .
$$

For $j=n$, we use $\Lambda(u) \leq 2|u|^{\gamma}$ and Stirling's formula to derive

$$
\left\{\int_{-\infty}^{\infty} \Lambda^{n}\left(\frac{y_{k_{n}}}{\sigma_{n}(\bar{t})} z\right) e^{-\frac{1}{2} z^{2}} d z\right\}^{1 / n} \leq A_{\overline{8.17} n^{\gamma}}\left(\frac{\|y\|}{\sigma_{n}(\bar{t})}\right)^{\gamma} .
$$

Hence, it follows from (8.10), (8.14), (8.15), (8.16) and (8.17) that

$$
\begin{gathered}
\max _{\bar{k} \in\{1, \ldots, d\}^{n}} \int_{F^{n}} \mathscr{M}(\bar{k}, \bar{t}) \mu^{n}(d \bar{t}) \leq \int_{F^{n}} \frac{A \overline{8.18}\|y\|^{\gamma}}{\left[\operatorname{det} \operatorname{Cov}\left(B_{1}\left(t^{1}\right), \ldots, B_{1}\left(t^{n-1}\right)\right)\right]^{d / 2}} \\
\times \frac{1}{\sigma_{n}^{d+\gamma}(\bar{t})} \prod_{j=1}^{n-1}\left\{\int_{-\infty}^{\infty} \Lambda^{n}\left(\frac{\|y\|}{\sigma_{j}(\bar{t})} z\right) e^{-\frac{1}{2} z^{2}} d z\right\}^{1 / n} \mu^{n}(d \bar{t}),
\end{gathered}
$$

where $A_{8.18}$ is a constant depending on $d, \gamma$ and $n$ only. We can estimate the preceding integral iteratively by integrating in the order $\mu\left(d t^{n}\right), \mu\left(d t^{n-1}\right), \ldots, \mu\left(d t^{1}\right)$.

Let $t^{1}, \ldots, t^{n-1} \in F$ be fixed points such that $t_{\ell}^{j}(1 \leq j \leq n-1,1 \leq \ell \leq N)$ are distinct. We consider the integral

$$
\mathscr{N}=\int_{F} \frac{1}{\sigma_{n}^{d+\gamma}(\bar{t})} \prod_{j=1}^{n-1}\left\{\int_{-\infty}^{\infty} \Lambda^{n}\left(\frac{\|y\|}{\sigma_{j}(\bar{t})} z\right) e^{-\frac{1}{2} z^{2}} d z\right\}^{1 / n} \mu\left(d t^{n}\right) .
$$

It follows from Proposition 4.2 that for every $1 \leq j \leq n$,

$$
\sigma_{j}^{2}(\bar{t}) \geq \frac{a^{N-1}}{2} \sum_{\ell=1}^{N} \min _{i \neq j}\left|t_{\ell}^{i}-t_{\ell}^{j}\right| \text {. }
$$


In order to estimate the sum in (8.20) as a function of $t^{n}$, we introduce $N$ permutations $\Gamma_{1}, \ldots, \Gamma_{N}$ of $\{1, \ldots, n-1\}$ such that for every $\ell=1, \ldots, N$,

$$
t_{\ell}^{\Gamma_{\ell}(1)}<t_{\ell}^{\Gamma_{\ell}(2)}<\ldots<t_{\ell}^{\Gamma_{\ell}(n-1)} .
$$

For convenience, we denote $t_{\ell}^{\Gamma_{\ell}(0)}=a$ and $t_{\ell}^{\Gamma_{\ell}(n)}=b$ for all $1 \leq \ell \leq N$.

For every $\left(i_{1}, \ldots, i_{N}\right) \in\{1, \ldots, n-1\}^{N}$, let $\tau_{i_{1}, \ldots, i_{N}}=\left(t_{1}^{\Gamma_{1}\left(i_{1}\right)}, \ldots, t_{N}^{\Gamma_{N}\left(i_{N}\right)}\right)$ be the "center" of the rectangle

$$
I_{i_{1}, \ldots, i_{N}}=\prod_{\ell=1}^{N}\left[t_{\ell}^{\Gamma_{\ell}\left(i_{\ell}\right)}-\frac{1}{2}\left(t_{\ell}^{\Gamma_{\ell}\left(i_{\ell}\right)}-t_{\ell}^{\Gamma_{\ell}\left(i_{\ell}-1\right)}\right), t_{\ell}^{\Gamma_{\ell}\left(i_{\ell}\right)}+\frac{1}{2}\left(t_{\ell}^{\Gamma_{\ell}\left(i_{\ell}+1\right)}-t_{\ell}^{\Gamma_{\ell}\left(i_{\ell}\right)}\right)\right)
$$

with the convention that the left-end point of the interval is $a$ whenever $i_{\ell}=1$; and the interval is closed and its right-end is $b$ whenever $i_{\ell}=n-1$. Thus the rectangles $\left\{I_{i_{1}, \ldots, i_{N}}\right\}$ form a partition of $[a, b]^{N}$.

For every $t^{n} \in F$, let $I_{i_{1}, \ldots, i_{N}}$ be the unique rectangle containing $t^{n}$. Then (8.20) yields the following estimate:

$$
\sigma_{n}^{2}(\bar{t}) \geq \frac{a^{N-1}}{2} \sum_{\ell=1}^{N}\left|t_{\ell}^{n}-t_{\ell}^{\Gamma_{\ell}\left(i_{\ell}\right)}\right| \geq A_{\overline{8.23}}|| t^{n}-\tau_{i_{1}, \ldots, i_{N}} \| .
$$

For every $j=1, \ldots, n-1$, we say that $I_{i_{1}, \ldots, i_{N}}$ cannot see $t^{j}$ from direction $\ell$ if

$$
t_{\ell}^{j} \notin\left[t_{\ell}^{\Gamma_{\ell}\left(i_{\ell}\right)}-\frac{1}{2}\left(t_{\ell}^{\Gamma_{\ell}\left(i_{\ell}\right)}-t_{\ell}^{\Gamma_{\ell}\left(i_{\ell}-1\right)}\right), t_{\ell}^{\Gamma_{\ell}\left(i_{\ell}\right)}+\frac{1}{2}\left(t_{\ell}^{\Gamma_{\ell}\left(i_{\ell}+1\right)}-t_{\ell}^{\Gamma_{\ell}\left(i_{\ell}\right)}\right)\right] .
$$

We emphasize that if $I_{i_{1}, \ldots, i_{N}}$ cannot see $t^{j}$ from all $N$ directions, then

$$
\left|t_{\ell}^{j}-t_{\ell}^{n}\right| \geq \frac{1}{2} \min _{i \neq j, n}\left|t_{\ell}^{j}-t_{\ell}^{i}\right| \quad \text { for all } 1 \leq \ell \leq N .
$$

Thus $t^{n}$ does not contribute to the sum in (8.20). More precisely, the latter means that

$$
\sigma_{j}^{2}(\bar{t}) \geq \frac{a^{N-1}}{4} \sum_{\ell=1}^{N} \min _{i \neq j, n}\left|t_{\ell}^{j}-t_{\ell}^{i}\right| .
$$

The right hand side of (8.26) only depends on $t^{1}, \ldots, t^{n-1}$, which will be denoted by $\tilde{\sigma}_{j}^{2}(\bar{t})$. Hence we have

$$
\int_{-\infty}^{\infty} \Lambda^{n}\left(\frac{\|y\|}{\sigma_{j}(\bar{t})} z\right) e^{-\frac{1}{2} z^{2}} d z \leq \int_{-\infty}^{\infty} \Lambda^{n}\left(\frac{\|y\|}{\tilde{\sigma}_{j}(\bar{t})} z\right) e^{-\frac{1}{2} z^{2}} d z
$$

If $I_{i_{1}, \ldots, i_{N}}$ sees $t^{j}$ from a direction, then, except in the special case $t^{j}=\tau_{i_{1}, \ldots, i_{N}}$, it is impossible to control $\sigma_{j}^{2}(\bar{t})$ from below as in (8.23) and (8.26) [recall that $B$ is not LND with respect to $\left.\mathrm{E}(B(u)-B(v))^{2}\right]$. We say that $t^{j}$ is a "bad point" for $I_{i_{1}, \ldots, i_{N}}$. In this case, we use the inequality $\Lambda(u) \leq 2$ to derive

$$
\int_{-\infty}^{\infty} \Lambda^{n}\left(\frac{\|y\|}{\sigma_{j}(\bar{t})} z\right) e^{-\frac{1}{2} z^{2}} d z \leq 2^{n} \sqrt{2 \pi}
$$

It is important to note that, because of (8.21), the rectangle $I_{i_{1}, \ldots, i_{N}}$ can only have at most $N$ bad points $t^{j}(1 \leq j \leq n-1)$, i.e., at most one in each direction. 
It follows from (8.23), (8.27) and (8.28) that

$$
\begin{aligned}
& \int_{I_{i_{1}, \ldots, i_{N}}} \frac{1}{\sigma_{n}^{d+\gamma}(\bar{t})} \prod_{j=1}^{n-1}\left\{\int_{-\infty}^{\infty} \Lambda^{n}\left(\frac{\|y\|}{\sigma_{j}(\bar{t})} z\right) e^{-\frac{1}{2} z^{2}} d z\right\}^{1 / n} \mu\left(d t^{n}\right) \\
& \leq A \int_{I_{i_{1}, \ldots, i_{N}}} \frac{\mu\left(d t^{n}\right)}{\| t^{n}-\tau_{i_{1}, \ldots, i_{N} \|^{(d+\gamma) / 2}}^{(d)}}\left\{\int_{-\infty}^{\infty} \Lambda^{n}\left(\frac{\|y\|}{\tilde{\sigma}_{j}(\bar{t})} z\right) e^{-\frac{1}{2} z^{2}} d z\right\}^{1 / n} \\
& \quad \times \prod_{j \notin \Theta_{i_{1}, \ldots, i_{N}}}\left\{\int_{j \notin \Theta_{i_{1}, \ldots, i_{N}}}^{\infty}\left\{\Lambda_{-\infty}^{n}\left(\frac{\|y\|}{\tilde{\sigma}_{j}(\bar{t})} z\right) e^{-\frac{1}{2} z^{2}} d z\right\}^{1 / n},\right.
\end{aligned}
$$

where $\Theta_{i_{1}, \ldots, i_{N}}=\left\{1 \leq j \leq n-1: t^{j}\right.$ is a bad point for $\left.I_{i_{1}, \ldots, i_{N}}\right\}$ and the last inequality follows from (8.2). Recall that the cardinality of $\Theta_{i_{1}, \ldots, i_{N}}$ is at most $N$, and $\Theta_{i_{1}, \ldots, i_{N}}$ may be the same for different values of $\left(i_{1}, \ldots, i_{N}\right)$.

Summing (8.29) over all $\left(i_{1}, \ldots, i_{N}\right) \in\{1, \ldots, n-1\}^{N}$ and regrouping $\Theta_{i_{1}, \ldots, i_{N}}$, we derive that the integral $\mathscr{N}$ is bounded above by

$$
\begin{aligned}
& A \overline{8.29} \sum_{i_{1}, \ldots, i_{N}} \prod_{j \notin \Theta_{i_{1}, \ldots, i_{N}}}\left\{\int_{-\infty}^{\infty} \Lambda^{n}\left(\frac{\|y\|}{\tilde{\sigma}_{j}(\bar{t})} z\right) e^{-\frac{1}{2} z^{2}} d z\right\}^{1 / n} \\
& \leq A_{\overline{8.30}} \sum_{\Theta} \prod_{j \notin \Theta}\left\{\int_{-\infty}^{\infty} \Lambda^{n}\left(\frac{\|y\|}{\tilde{\sigma}_{j}(\bar{t})} z\right) e^{-\frac{1}{2} z^{2}} d z\right\}^{1 / n},
\end{aligned}
$$

where the last summation is taken over all $\Theta \subset\{1, \ldots, n-1\}$ with $\# \Theta \leq N$ and $A_{8.30}$ depends on $(d, a, b, N, \gamma, n)$ only. Note that the number of terms in the last sum is at most $(n-1)^{N}$.

Put (8.30) into (8.18) to obtain

$$
\begin{aligned}
& \max _{\bar{k} \in\{1, \ldots, d\}^{n}} \int_{F^{n}} \mathscr{M}(\bar{k}, \bar{t}) \mu^{n}(d \bar{t}) \\
& \leq \sum_{\Theta} \int_{F^{n-1}} \frac{A \overline{8.31}\|y\|^{\gamma}}{\left[\operatorname{det} \operatorname{Cov}\left(B_{1}\left(t^{1}\right), \ldots, B_{1}\left(t^{n-1}\right)\right)\right]^{d / 2}} \\
& \quad \times \prod_{j \notin \Theta}\left\{\int_{-\infty}^{\infty} \Lambda^{n}\left(\frac{\|y\|}{\tilde{\sigma}_{j}(\bar{t})} z\right) e^{-\frac{1}{2} z^{2}} d z\right\}^{1 / n} \mu^{n-1}(d \bar{t}) .
\end{aligned}
$$

We observe that:

(i) Increasing the number of elements in $\Theta$ changes only the integrals in (8.31) by a constant factor [recall (8.28)]; and

(ii) $\operatorname{det} \operatorname{Cov}\left(B_{1}\left(t^{1}\right), \ldots, B_{1}\left(t^{n-1}\right)\right)$ is symmetric in $t^{1}, \ldots, t^{n-1}$. 
Based on these observations we can deduce that the following is valid uniformly for all $\bar{k} \in\{1, \ldots, d\}^{n}$ :

$$
\begin{aligned}
\int_{F^{n}} \mathscr{M}(\bar{k}, \bar{t}) \mu^{n}(d \bar{t}) \leq & \int_{F^{n-1}} \frac{A \overline{8.32}\|y\|^{\gamma}}{\left[\operatorname{det} \operatorname{Cov}\left(B_{1}\left(t^{1}\right), \ldots, B_{1}\left(t^{n-1}\right)\right)\right]^{d / 2}} \\
& \times \prod_{j=N+1}^{n-1}\left\{\int_{-\infty}^{\infty} \Lambda^{n}\left(\frac{\|y\|}{\tilde{\sigma}_{j}(\bar{t})} z\right) e^{-\frac{1}{2} z^{2}} d z\right\}^{1 / n} \mu^{n-1}(d \bar{t}) .
\end{aligned}
$$

Here, $A_{8.32}$ is a constant depending only on $(d, a, b, N, \gamma, n)$, and the last product $\prod_{j=N+1}^{n-1} \cdots$ can be replaced by 1 if $n \leq N+1$.

By repeating the preceding argument and integrating $\mu\left(d t^{n-1}\right), \ldots, \mu\left(d t^{1}\right)$ iteratively and by (8.14), we obtain

$$
\mathrm{E}\left[\left(l_{\mu}(x)-l_{\mu}(x+y)\right)^{n}\right] \leq A_{8.33}\|y\|^{\left\llcorner\frac{n}{N+1}\right\rfloor \gamma},
$$

where $A$ is a constant depending on $(d, a, b, N, \gamma, n)$ only and $\|y\|^{\left\lfloor\frac{n}{N+1}\right\rfloor \gamma}$ comes from the first $\left\lfloor\frac{n}{N+1}\right\rfloor$-steps of integration. This, together with a multiparameter version of the Kolmogorov continuity theorem [15, Theorem 2.5.1, p. 165] proves Equation (8.6), but where $u$ and $v$ are restricted to a given compact set. It follows readily that we can construct a version of $\left\{l_{\mu}(x)\right\}_{x \in \mathbf{R}^{d}}$ that is Hölder-continuous with parameter $<\gamma /(N+1)$ on all compact subsets of $\mathbf{R}^{d}$. But $x \mapsto l_{\mu}(x)$ is, by its definition and the continuity of $B$, a compact-support function because $\mu$ lives on the compact set $F \subset \mathbf{R}_{+}^{N}$. With a bit of measure theory, this completes our proof.

8.4. Continuity in the rotation variable. We hold $F$ and $\mu$ fixed as in the previous subsection. Let $\mathscr{R}$ denote the collection of all $N$-by- $N$ rotation matrices that leave $F$ in $\mathbf{R}_{+}^{N}$; i.e., $\theta \in \mathscr{R}$ if and only if $\theta$ is a rotation matrix such that $\theta F \subset \mathbf{R}_{+}^{N}$. We endow all square matrices with their $\ell^{2}$ matrix-norm; i.e., for all $N$-by- $N$ matrices $M$,

$$
\|M\|=\sup _{x \in \mathbf{R}^{N}:\|x\|=1}(x \cdot M x)^{1 / 2}=\sup _{x \in \mathbf{R}^{N}:\|x\|=1}\|M x\| .
$$

Define

$$
\mu_{\theta}(G)=\mu\left(\theta^{-1} G\right) \quad{ }^{\forall} \text { Borel sets } G \subset \mathbf{R}_{+}^{N}
$$

Manifestly, $\mu_{\theta}$ is in $\mathscr{P}(F)$, and satisfies (8.2) where $\mu$ is now replaced by $\mu_{\theta}$, but the constant $A_{8.2}$ remains unchanged. Thanks to Theorem 8.1, $l_{\mu_{\theta}}$ is a.s. Hölder continuous for each $\theta \in \mathscr{R}$. We now prove that there is a continuous version of $(x, \theta) \mapsto l_{\mu_{\theta}}(x)$.

Throughout, we define

$$
\tau(F)=\frac{\operatorname{dim}_{\mathcal{H}} F-\frac{d}{2}}{2 \operatorname{dim}_{\mathcal{H}} F+N(d+1)+1} .
$$

Theorem 8.4. Let $F$ be a compact set in $\mathbf{R}_{+}^{N}$ that satisfies (6.1), and suppose $\mu \in \mathscr{P}(F)$ satisfies (8.2). For every even integer $n \geq 2$ and every $0<\delta<\tau(F)$,

$$
\mathrm{E}\left[\sup _{\substack{\theta, \rho \in \mathscr{R}: \\ \theta \neq \rho}} \frac{\left|l_{\mu_{\theta}}(x)-l_{\mu_{\rho}}(x)\right|^{n}}{\|\theta-\rho\|^{n \delta}}\right]<\infty .
$$


Consequently, for all $0<\eta<\min \left(1, \frac{2}{N+1}\left(\operatorname{dim}_{\mathcal{H}} F-\frac{d}{2}\right)\right)$ and $0<\delta<\tau(F)$, there exists a version of $\left\{l_{\mu_{\theta}}(x)\right\}_{x \in \mathbf{R}^{d} ; \theta \in \mathscr{R}}$ that is uniformly Hölder-continuous: In $x \in \mathbf{R}^{d}$ with index $\eta$, and in $\theta \in \mathscr{R}$ with index $\delta$.

Proof. There exists a function $\psi: \mathbf{R}^{d} \rightarrow \mathbf{R}_{+}$that has the following properties:

- There exists a finite constant $c_{\psi}$ such that for all $x, y \in \mathbf{R}^{d},|\psi(x)-\psi(y)| \leq$ $c_{\psi}\|x-y\|$

- $\psi(x) \geq 0$ and $\int_{\mathbf{R}^{d}} \psi(x) d x=1$;

- $\psi(x)>0$ if $\|x\| \leq \frac{1}{2}$, whereas $\psi(x)=0$ if $\|x\| \geq 1$.

For all $\varepsilon>0$, define

$$
\psi_{\varepsilon}(x)=\varepsilon^{-d} \psi(x / \varepsilon) \quad{ }^{\forall} x \in \mathbf{R}^{d} .
$$

First of all, note that for all $a \in \mathbf{R}^{d}, \theta \in \mathscr{R}$, and $\varepsilon>0$, the following holds a.s.:

$$
\begin{aligned}
\left|\int_{F} \psi_{\varepsilon}(B(s)-a) \mu_{\theta}(d s)-l_{\mu_{\theta}}(a)\right| & =\left|\int_{\mathbf{R}^{d}} \psi_{\varepsilon}(x-a) l_{\mu_{\theta}}(x) d x-l_{\mu_{\theta}}(a)\right| \\
& \leq \int_{\mathbf{R}^{d}} \psi_{\varepsilon}(x-a)\left|l_{\mu_{\theta}}(x)-l_{\mu_{\theta}}(a)\right| d x \\
& \leq \sup _{x \in \mathbf{R}^{d}:\|x-a\| \leq \varepsilon}\left|l_{\mu_{\theta}}(x)-l_{\mu_{\theta}}(a)\right| \\
& =\Omega_{\theta}(\varepsilon) .
\end{aligned}
$$

[Justification: The first line follows from (8.5); second from the fact that $\psi_{\varepsilon}$ integrates to one; and third from the fact that $\psi_{\varepsilon}$ is supported on the centered ball of radius $\varepsilon$.] Furthermore, $\psi_{\varepsilon}$ is Lipschitz-continuous with Lipschitz-constant $c_{\psi} \varepsilon^{-(d+1)}$. Therefore, for all $\theta, \rho \in \mathscr{R}$, all $a \in \mathbf{R}^{d}$, and all $\varepsilon>0$, with probability one,

$$
\begin{aligned}
& \left|\int_{F} \psi_{\varepsilon}(B(s)-a) \mu_{\theta}(d s)-\int_{F} \psi_{\varepsilon}(B(s)-a) \mu_{\rho}(d s)\right| \\
& =\left|\int_{F}\left[\psi_{\varepsilon}(B(\theta s)-a)-\psi_{\varepsilon}(B(\rho s)-a)\right] \mu(d s)\right| \\
& \leq c_{\psi} \varepsilon^{-(d+1)} \sup _{s \in F}|| B(\theta s)-B(\rho s)|| .
\end{aligned}
$$

Combine (8.39) and (8.40) to deduce that a.s.:

$$
\left|l_{\mu_{\theta}}(a)-l_{\mu_{\rho}}(a)\right| \leq \Omega_{\theta}(\varepsilon)+\Omega_{\rho}(\varepsilon)+c_{\psi} \varepsilon^{-(d+1)} \sup _{s \in F} \| B(\theta s)-B(\rho s)|| .
$$

One can use this to directly construct a continuous version of these local times. However, we will outline a more standard approach.

By continuity (Theorem 8.1), (8.41) holds simultaneously for all $a \in \mathbf{R}^{d}$. Therefore, by Minkowski's inequality, for all even integers $n \geq 2$,

$$
\begin{aligned}
& \left(\mathrm{E}\left[\sup _{a \in \mathbf{R}^{d}}\left|l_{\mu_{\theta}}(a)-l_{\mu_{\rho}}(a)\right|^{n}\right]\right)^{1 / n} \\
& \leq\left\|\Omega_{\theta}(\varepsilon)\right\|_{L^{n}(\mathrm{P})}+\left\|\Omega_{\rho}(\varepsilon)\right\|_{L^{n}(\mathrm{P})} \\
& \quad+c_{\psi} \varepsilon^{-(d+1)}\left(\mathrm{E}\left[\sup _{s \in F} \| B(\theta s)-B(\rho s)||^{n}\right]\right)^{1 / n} .
\end{aligned}
$$


Fix some positive $\eta<\min \left(1, \frac{2}{N+1}\left(\operatorname{dim}_{\mathcal{H}} F-\frac{d}{2}\right)\right)$ to see that the first two terms are each bounded above by $A \overline{8.6}^{\eta}$; see (8.6). Because $F$ is compact, standard Kolmogorov-continuity estimates show that the third term is at most a universal constant [depending only on $(d, N, a, b)$ ] times $\varepsilon^{-(d+1)}\|\theta-\rho\|^{1 / 2}$; for example, see Exercise 7 of [15, p. 176]. Optimize the resulting inequality over all $\varepsilon$ to obtain (8.37). The remainder of the proof follows from a multiparameter version of the Kolmogorov continuity theorem [15, Theorem 2.5.1, p. 165].

8.5. The remainder of the proof of Theorem 1.4. We are ready to assemble the pieces that complete the proof of Theorem 1.4. Throughout, we may, and will, assume that $\left\{l_{\mu_{\theta}}(x)\right\}_{x \in \mathbf{R}^{d} ; \theta \in \mathscr{R}}$ is continuous (Theorem 8.4).

According to (8.5), we have

$$
\int_{\mathbf{R}^{d}} l_{\mu_{\theta}}(x) d x=1 \quad{ }^{\forall} \theta \in \mathscr{R} .
$$

This uses the continuity of local times and $B$, as well as the compactness of $F$. Note that we have stopped writing "a.s." because from now on, there is only one null-set left, and so it can be ignored.

Continuity insures that for every $\theta \in \mathscr{R}$ there exists an open ball $J_{\theta} \subset \mathbf{R}^{d}$ such that for all $x \in J_{\theta}, l_{\mu_{\theta}}(x)>0$. This is enough to prove that for all $\theta \in \mathscr{R}, B(\theta F)$ has interior-points: Any $x \in J_{\theta}$ is an interior-point of $B(\theta F)$.

In order to prove the stronger assertion of the theorem, we need to refine the $J_{\theta}$ 's slightly.

Due to continuity, for every $\theta \in \mathscr{R}$ we can find an open ball $K_{\theta} \subseteq J_{\theta} \subset \mathbf{R}^{d}$ and an open ball $V_{\theta} \subset \mathscr{R}$ such that for all $\rho \in V_{\theta}$ and all $x \in K_{\theta}, l_{\mu_{\rho}}(x)>0$. Now $\left\{V_{\theta}\right\}_{\theta \in \mathscr{R}}$ is an open cover of $\mathscr{R}$, where the latter is viewed as a closed subset of $\mathfrak{H}_{N}$ - the rotation group acting on $\mathbf{R}^{N}$. Because $\mathfrak{H}_{N}$ is compact 22, Section 65, p. 489], so is $\mathscr{R}$. It follows that there is a finite subcover $\left\{V_{\theta(j)}\right\}_{j=1}^{m}$ of $\mathscr{R}$; it has the property that for every $\rho \in V_{\theta(j)}$ and all $x \in K_{\theta(j)}, l_{\mu_{\rho}}(x)>0$. Let $\zeta_{j}$ denote the midpoint of the interval $K_{\theta(j)}$ to deduce the theorem.

\section{An ARITHMEtic PRoperty of Brownian motion}

We conclude this paper by proving an arithmetic result about Brownian motion. Henceforth, $\{X(t)\}_{t \geq 0}$ denotes $d$-dimensional Brownian motion, and $F$ a fixed compact subset of $\mathbf{R}_{+}$.

Choose and fix an integer $N \geq 1$, and $N$ non-zero real numbers $r_{1}, \ldots, r_{N}$, once and for all. Define the [inhomogeneous] $N$-fold Brownian self-intersection field [23]:

$$
S(t)=\sum_{j=1}^{N} r_{j} X\left(t_{j}\right) \quad{ }^{\forall} t \in \mathbf{R}_{+}^{N} .
$$

Next is a refinement to Theorem 1 of [19]; see also [13].

Theorem 9.1. Suppose $G_{1}, \ldots, G_{N}$ are compact subsets of $\mathbf{R}_{+}$, and let $G=$ $G_{1} \times \cdots \times G_{N}$. If $\operatorname{dim}_{\mathcal{H}} G>d / 2$, then $S(G)$ a.s. has interior-points. Moreover, $\operatorname{Cap}_{d / 2}(G)=0$ if and only if $S(G)$ a.s. has zero Lebesgue measure.

Before we prove this, we make some observations. 
Remark 9.2. Note the elementary bounds,

$$
\sum_{j=1}^{N} \operatorname{dim}_{\mathcal{H}}\left(G_{j}\right) \leq \operatorname{dim}_{\mathcal{H}}\left(G_{1} \times \cdots \times G_{N}\right) \leq \sum_{j=1}^{N} \operatorname{dim}_{\mathcal{P}}\left(G_{j}\right),
$$

where $\operatorname{dim}_{\mathcal{P}}$ denotes the packing dimension. Therefore, Theorem 9.1 implies that:

- If $\operatorname{dim}_{\mathcal{H}}(F)>d /(2 N)$, then $X(F) \oplus \cdots \oplus X(F)$ contains interior-points a.s.

- If $\operatorname{dim}_{\mathcal{P}}(F)<d /(2 N)$, then $X(F) \oplus \cdots \oplus X(F)$ is Lebesgue-null a.s.

The first item is a minor generalization of Theorems 2-4 of [19]; the second item slightly improves upon Comment (2) of [19, p. 459] who derives this assertion with upper Minkowski dimension in place of packing dimension.

To prove Theorem 9.1 we may - and will — assume without loss of generality that $G_{i} \subset\left[a_{i}, b_{i}\right]$ and

$$
0<a_{1}<b_{1}<a_{2}<b_{2}<\cdots<a_{N}<b_{N} .
$$

Similar reductions have been made earlier by [19] and [13. To simplify the formulation of Proposition 9.3. we assume further that for all relevant integers $i$ and $j$, $\left(b_{i}-a_{i}\right) \leq\left(a_{j+1}-b_{j}\right)$.

Thanks to Lemma 4.3, we can deduce

Proposition 9.3. Suppose $\left\{G_{i}\right\}_{i=1}^{N}$ are compact subsets of $\mathbf{R}_{+}$that satisfy the preceding conditions. Then the process $S$ is sectorially $L N D$ on $G:=G_{1} \times \cdots \times G_{N}$. In fact, for all $u, t^{1}, \ldots, t^{n} \in \mathbf{R}_{+}^{N}$,

$$
\operatorname{Var}\left(S_{1}(u) \mid S_{1}\left(t^{1}\right), \ldots, S_{1}\left(t^{n}\right)\right) \geq \frac{\min _{1 \leq k \leq N} r_{k}^{2}}{2 N} \sum_{k=1}^{N} \min _{1 \leq j \leq n}\left|u_{k}-t_{k}^{j}\right| .
$$

Proof. Let $u=\left(u_{1}, \ldots, u_{N}\right)$. It follows from Lemma 4.3 that for each $1 \leq k \leq N$

$$
\begin{aligned}
\operatorname{Var}\left(S_{1}(u) \mid S_{1}\left(t^{1}\right), \ldots, S_{1}\left(t^{n}\right)\right) & \geq \operatorname{Var}\left(X\left(u_{k}\right) \mid X\left(t_{\ell}^{j}\right), \forall j, \ell ; X\left(u_{i}\right), i \neq k\right) \\
& \geq \frac{r_{k}^{2}}{2} \min _{1 \leq j \leq n}\left|u_{k}-t_{k}^{j}\right| .
\end{aligned}
$$

Summing over $k$ yields (9.4).

Proof of Theorem 9.1. We go through the proof of Theorem 8.1, but use Proposition 9.3 in place of Proposition 4.2 everywhere. This immediately proves that $S$ has a continuous local time on $G$ when $\operatorname{dim}_{\mathcal{H}} G>d / 2$. Therefore, $S(G)$ has interior-points almost surely.

For the capacity condition, we simply compare $S$ to an additive Brownian motion. This is achieved by combining the proof of Theorem 6.1 of [17] with Theorem 4.2 in the same paper.

\section{A FinAL REMARK}

Consider an arbitrary centered Gaussian random field $\{G(t)\}_{t \in \mathbf{R}_{+}^{N}}$, a compact set $F$ in $\mathbf{R}_{+}^{N}$ that satisfies (6.1) and $\mu \in \mathscr{P}(F)$ satisfies (8.2) with $\gamma \in(0,1)$. An inspection of our proof of Theorem 8.1 shows that everything up to and including (8.19) is valid as long as $G$ has i.i.d. coordinate-processes. The rest of the proof depends crucially on whether one can derive an appropriate upper bound for (8.19). 
If we apply the inequality $\Lambda(u) \leq 2|u|^{\gamma}$ to 8.15 for all $j=1, \ldots, n$, then for all $x, y \in \mathbf{R}^{d}$ and all even integers $n \geq 2$ we can find a finite constant $A_{10.1}=$ $A_{10.1}(d, a, b, \gamma, n)$ such that

$$
\begin{aligned}
& \mathrm{E}\left[\left(l_{\mu}(x)-l_{\mu}(x+y)\right)^{n}\right] \\
& \leq A_{10.1}\|y\|^{n \gamma} \int_{F^{n}} \frac{\prod_{j=1}^{n}\left[\operatorname{Var}\left(G_{1}\left(t^{j}\right) \mid\left\{G_{1}\left(t^{i}\right)\right\}_{i \neq j}\right)\right]^{-\gamma}}{\left[\operatorname{det} \operatorname{Cov}\left(G_{1}\left(t^{1}\right), \ldots, G_{1}\left(t^{n}\right)\right)\right]^{d / 2}} \mu^{n}(d \bar{t}) .
\end{aligned}
$$

Hence we have derived the following convenient result.

Theorem 10.1. Let $\{G(t)\}_{t \in \mathbf{R}_{+}^{N}}$ be an $N$-parameter centered Gaussian process in $\mathbf{R}^{d}$ that has i.i.d. continuous coordinate-processes. Choose and fix a compact set $F \subset \mathbf{R}_{+}^{N}$ such that $\sup _{t \in F} \operatorname{Var}\left(G_{1}(t)\right)<\infty$. Assume that there exists $\mu \in \mathscr{P}(F)$, an even integer $n>N$, and some $\gamma \in\left(\frac{N}{n}, 1\right)$ such that

$$
\int_{F^{n}} \frac{\prod_{j=1}^{n}\left[\operatorname{Var}\left(G_{1}\left(t^{j}\right) \mid\left\{G_{1}\left(t^{i}\right)\right\}_{i \neq j}\right)\right]^{-\gamma}}{\left[\operatorname{det} \operatorname{Cov}\left(G_{1}\left(t^{1}\right), \ldots, G_{1}\left(t^{n}\right)\right)\right]^{d / 2}} \mu^{n}(d \bar{t})<\infty .
$$

Then $\left\{l_{\mu}(x)\right\}_{x \in \mathbf{R}^{d}}$ has a modification which is Hölder continuous of any order $<\gamma$. Consequently, $G(F)$ has interior-points almost surely.

Remark 10.2. Condition (10.2) is satisfied by a large class of locally non-deterministic Gaussian random fields [24].

\section{ACKNOWLEDGMENTS}

We thank the referee for a careful reading of our paper, and for making thoughtful comments. These comments have led to several improvements in the exposition of this paper.

\section{REFERENCES}

[1] Robert J. Adler, The Geometry of Random Fields, Wiley, New York, 1981. MR0611857 (82h:60103)

[2] Correction to: "The uniform dimension of the level sets of a Brownian sheet" [Ann. Probab. 6 (1978), no. 3, 509-515], Ann. Probab. 8(5) (1980), 1001-1002. MR0600348 (82h:60148)

[3] , The uniform dimension of the level sets of a Brownian sheet, Ann. Probab. 6(3) (1978), 509-515. MR0490818 (80a:60102)

[4] R. Cairoli and John B. Walsh, Stochastic integrals in the plane, Acta Math. 134 (1977), 111-183. MR0420845(54:8857)

[5] Jack Cuzick and Johannes P. DuPreez, Joint continuity of Gaussian local times, Ann. Probab. 10(3) (1982), 810-817. MR0659550 (83f:60061)

[6] W. Ehm, Sample function properties of multiparameter stable processes, Z. Wahrsch. Verw. Gebiete 56(2) (1981), 195-228. MR0618272 (82g:60096)

[7] Kenneth Falconer, Fractal Geometry, John Wiley \& Sons Ltd., Chichester, 1990. MR.1102677 (92j:28008)

[8] Donald Geman and Joseph Horowitz, Occupation densities, Ann. Probab. 8(1) (1980), 1-67. MR0556414 (81b:60076)

[9] John Hawkes, Local properties of some Gaussian processes, Z. Wahrscheinlichkeitstheorie und Verw. Gebiete 40(4) (1977), 309-315. MR0458559(56:16759)

[10] Jean-Pierre Kahane, Some Random Series of Functions, Second Edition, Cambridge University Press, Cambridge, 1985a. MR0833073 (87m:60119)

[11] _ Ensembles aléatoires et dimensions, In: Recent progress in Fourier analysis (El Escorial, 1983), 65-121, North-Holland, Amsterdam (1985b). MR0848143 (87k:60110) 
[12] _ Ensembles parfaits et processus de Lévy, Period. Math. Hungar. 2 (1972), 49-59. MR0329050 (48:7392)

[13] Robert Kaufman, On the sum of two Brownian paths, Studia. Math. LXV (1979), 51-54. MR0554540 (80k:60100)

[14] Fourier analysis and paths of Brownian motion, Bull. Soc. Math. France 103 (1975), 427-432. MR0397905 (53:1760)

[15] Davar Khoshnevisan, Multiparameter Processes: An Introduction to Random Fields, Springer, New York, 2002. MR,1914748(2004a:60003)

[16] _ Brownian sheet images and Bessel-Riesz capacity, Trans. Amer. Math. Soc. 351(7) (1999), 2607-2622. MR 1638246(2000e:60123)

[17] Davar Khoshnevisan and Yimin Xiao, Lévy Processes: Capacity and Hausdorff dimension, Ann. Probab. 33(3) (2005), 841-878. MR2135306 (2006d:60078)

[18] T. S. Mountford, A relation between Hausdorff dimension and a condition on time sets for the image by the Brownian sheet to possess interior-points, Bull. London Math. Soc. 21 (1989), 179-185. MR0976063 (89m:60094)

[19] _ An extension of a result of Kahane using Brownian local times of intersection, Stochastics 23(4) (1988), 449-464. MR0943815 (89m:60197)

[20] Yuval Peres, Probability on Trees: An Introductory Climb, Lectures on probability theory and statistics (Saint-Flour, 1997), 1999, pp. 193-280. MR.1746302 (2001c:60139)

[21] Lauren D. Pitt, Local times for Gaussian vector fields, Indiana Univ. Math. J. 27 (1978), 309-330. MR0471055 (57:10796)

[22] L. S. Pontryagin, Topological Groups, Translated from the second Russian edition by Arlen Brown, Gordon and Breach Science Publishers, Inc., New York, 1966. MR0201557 (34:1439)

[23] Robert L. Wolpert, Local time and a particle picture for Euclidean field theory, J. Funct. Anal. 30(3) (1978), 341-357. MR0518340 (80a:81082)

[24] Yimin Xiao, Hölder conditions for the local times and the Hausdorff measure of the level sets of Gaussian random fields, Probab. Theory Rel. Fields 109 (1997), 129-157. MR.1469923 (98m:60060)

Department of Mathematics, The University of Utah, 155 S. 1400 E., Salt Lake City, UTAн 84112-0090

E-mail address: davar@math.utah.edu

$U R L:$ http://www.math.utah.edu/ davar

Department of Statistics and Probability, A-413 Wells Hall, Michigan State UniVersity, East Lansing, Michigan 48824

E-mail address: xiao@stt.msu.edu

URL: http://www.stt.msu.edu/ xiaoyimi 